[4] N. Bourbaki, Fonctions d'une variable réelle, Paris 1949.

[5] - Espaces vectoriels topologiques, Paris 1966.

[6] - Variétés différentielles et analytiques (Résultats), Paris 1967.

[7] A. Grothendieck, Sur certains espaces de fonctions holomorphes, J. für reine u. angew. Math. 192 (1953), pp. 35-64.

[8] E. Hille and E. G. Philips, Functional analysis and semi-groups, Colloquium A. M.S., 1957.

[9] S. Mazur und W. Orlicz, Grundlegende Eigenschaften der polynomischen $O p$. erationen, Studia Math. 5 (1935), pp. 50-68, 179-189.

[10] Ph. Noverraz, Fonctions plurisousharmoniques et analytiques dans les espaces vectoriels topologiques complexes, Thèse, Paris 1969.

\section{Analytic functions in topological vector spaces}

\section{by}

JACEK B O CH N A K and JÓZEF S I C I A K (Kraków)

Contents

Introduction

1. Integral of a continuous function of a real variable with ralues in a locally convex space ... . . . . . . . . .

2. Weakly differentiable functions of one scalar variable ... 3. Analytic functions from $C^{n}$ to $F$. . . . . . . . . . . .

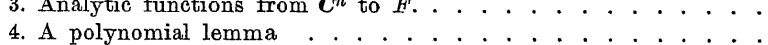

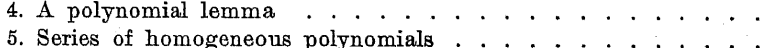

5. Series of homogeneous polynomials

7. Analytic functions in topological vector spaces over $\boldsymbol{R}$. . . .

8. Entire functions . . . . . . . . . . . .

9. List of problems . . . . . . . . . . . . . . .

References . . . . . . . . . . . . . . . . . .

Introduction. The aim of this paper is to give a unified and selfcontained exposition of basic concepts and facts concerning analytic functions defined in open subsets of a complex or real topological vector space $E$ with values in a locally convex topological vector space $F$. As a special case we get the theory developed in [2] and [11] for Banach spaces.

In the present article results of our previous paper [6] on polynomials and multilinear functions are essentially used.

We hope that our exposition may be useful for further study of the theory of analytic functions in topological vector spaces. This theory may be treated in natural way as a branch of non-linear functional analysis and deserves further developement.

In this paper we always assume that $E$ is a topological Hausdorff vector space, $F$ is locally convex and sequentially complete. However, in many places additional assumptions are necessary. Generally speaking, all the results are valid if $E$ is a Baire space (sometimes Baire and metrizable) - in the complex case, or if $E$ and $F^{\prime}$ (the topological dual of $F$ ) are Baire spaces - in the real case. 
In order to make the exposition selfcontained we introduce in Section 1 a notion of integral of continuous function of a real variable with values in $F$.

The main result of Section 2 is Lemma 2.1 which permits to imply that a function has the derivative of order $n$ if it has the weak derivative of order $n+1$. This lemma along with the Polynomial Lemma of Section 4 are basic for the greater part of further results.

Similarly, as in the case when $F$ is a Banach space, the most important theorems of the classical theory may be generalized almost literally to the case of analytic functions from $C^{n}$ to $F$, where $F$ is a locally convex, sequentially - complete t.v.s. over $\boldsymbol{C}$ (see Section 3).

In Section 5 we develop a theory of series $\sum_{n=0}^{\infty} f_{n}$, where $f_{n}$ is a homogeneous polynomial of degree $n$ from $E$ to $F$. Here the main results are: $1^{0}$ if the domain of convergence $H$ of the series $\sum_{n=0}^{\infty} f_{n}$ is not empty, then $H$ is a balanced neighborhood of $0 \in E$, and $f=\sum_{n=0}^{\infty} f_{n}^{n=0}$ is analytic (or $G$-analytic) in $H, 2^{\circ}$ if $E$ is a real space, then the complexified series $\sum_{n=0}^{\infty} \tilde{f}_{n}$ converges in an open set $\tilde{H} \subset \tilde{E}$ such that $H \subset \tilde{H}$. This permits to treat real analytic functions as restrictions of some complex analytic functions (see Theorems 7.1 and 7.2 )

Main theorems of this paper are presented in Sections 6 and 7. Here we give several necessary and sufficient conditions that a function $f: U \rightarrow F$ ( $U$ is an open subset of $E$ ) be analytic. We try to elucidate interrelations between analyticity, $G$-analyticity and weak-analyticity. In Section 6 we give also generalizations of the classical theorems on convergent sequences of analytic functions (Weierstrass theorem, Vitali theorem).

The most interesting result of Section 7 seems to be Theorem 7.5, which gives a characterization of real analytic functions. This theorem permits to generalize classical theorems due to Bernstein, due to Pringsheim-Boas and a theorem of Malgrange.

The paper is closed by Section 8 , devoted to some results on entire mappings $f: E \rightarrow F$. The main result: $f$ is entire if and only if $f \mid V$ is entire for every affine line $V \subset E$.

While preparing this paper we have been much inspired by [2], [11] and [18]. The references inclosed are far from complete. For further information we refer the reader to [11] and to Seminars of Lelong, [14], [15].

In the sequel $K$ denotes either the field of complex numbers $\boldsymbol{C}$ or the field of real numbers $\boldsymbol{R}$. Letters $E, F$ (with indices eventually) will denote Hausdorff topological vector spaces over $K$ (shortly t.v.s. over $K$ ). If the field is not strictly indicated the results are valid in both cases
$K=\boldsymbol{C}$ and $K=\boldsymbol{R}$. We always assume that t.v.s. $F$ is locally convex and $s$-oomplete (i.e. every. Cauchy sequence is convergent in $F$; comp. [6]). $\Gamma(F)$ denotes any filtrant set of seminorms determining the topology of $F . F^{\prime}$ is the vector space of all continuous linear forms $u: F \rightarrow K$. $\tilde{E}$ (resp. $\tilde{f}$ ) denotes the complexification of the t.v.s. $E$ (resp. of the homogeneous polynomial $f$ ) (comp. [6], § 2). $G^{\infty}(U)$ denotes the set of the functions of class $G^{\infty}$ defined on an open subset $U \subset E$, i.e. the set of functions having the Gateaux differentials $\delta_{x}^{n} f, n=1,2, \ldots$ at any point $x \in U$ (comp. $[6] ; \S 3$ ).

1. Integral of a continuous function of a real variable with values in a locally convex space.

LEMMa 1.1. Let $F$ be a locally convex s-complete t.v.s. over $K$. If $[a, b]$ denotes a compact segment of $\boldsymbol{R}$ and $f:[a, b] \rightarrow F$ is a continuous function, then the sequence

$$
I_{n}=\frac{b-a}{n} \sum_{k=1}^{n} f\left(a+\frac{k}{n}(b-a)\right), \quad n=1,2, \ldots,
$$

converges to an element $I \in \cdot F$.

Proof. Given $q \in \Gamma\left(F^{2}\right)$ and $\delta>0$, put

$$
\omega_{q}(\delta)=\sup \{q(f(x)-f(y)): x, y \in[a, b],|x-y| \leqslant \delta\} .
$$

Observe that

$$
q\left(I_{m}-I_{n}\right) \leqslant q\left(I_{m}-I_{m n}\right)+q\left(I_{n}-I_{m n}\right) \leqslant(b-a)\left(\omega_{q}\left(\frac{b-a}{m}\right)+\omega_{q}\left(\frac{b-a}{n}\right)\right)
$$

for $m, n=1,2, \ldots$ and $q \epsilon \Gamma(F)$. Since $F$ is $s$-complete, it follows that $\left\{I_{n}\right\}_{n \in N}$ is convergent to an element $I$ of $F$.

Defrintition 1.1. The limit $I$ of sequence (*) will be called the integrat of $f$ over $[a, b]$ and denoted by $\int_{a}^{b} f(t) d t$,

$$
\int_{a}^{b} f(t) d t=\lim _{n \rightarrow \infty} \frac{b-a}{n} \sum_{k=1}^{n} f\left(a+\frac{k}{n}(b-a)\right) .
$$

PRoposirion 1.1. Let $f, g$ be continuous functions from $[a, b]$ to $F$, and let $\alpha, \beta$ denote elements of $K$. Then

$$
\begin{aligned}
& \left(\mathrm{i}_{1}\right) \int_{a}^{b}(\alpha f+\beta g) d t=\alpha \int_{a}^{b} f d t+\beta \int_{a}^{b} g d t \\
& \left(\mathrm{i}_{2}\right) \cdot q(f f d t) \leqslant(b-a) M_{a}, M_{q}=\sup \{q \circ f(t): t \in[a, b]\}, q \in \Gamma(F)
\end{aligned}
$$


(i $\left.i_{3}\right)$ If functions $f_{n}:[a, b] \rightarrow F(n=1,2, \ldots)$ are continuous and $q \circ\left(f_{n}-f\right) \rightarrow 0$ uniformly on $[a, b]$, as $n \rightarrow \infty$, for every $q \in \Gamma(F)$, then

$$
\int_{a}^{b} f d t=\lim _{n \rightarrow \infty} \int_{a}^{b} f_{n} d t
$$

(i $\left.\mathbf{i}_{4}\right) u\left(\int_{a}^{b} f d t\right)=\int_{a}^{b} u \circ f d t, u \in F^{\prime}$;

(i. $\left.\mathrm{i}_{5}\right)$ If $\varphi:[c, d] \rightarrow[a, b]$ is a function of class $C^{1}$ such that $\varphi(c)$ $=a, \varphi(d)=b$, then

$$
\int_{a}^{b} f d t=\int_{c}^{a}(f \circ \varphi) \varphi^{\prime} d t
$$

Proof. $\left(i_{1}\right),\left(i_{2}\right),\left(i_{3}\right),\left(i_{4}\right)$ are obvious. Property $\left(i_{5}\right)$ is an immediate consequence of $\left(i_{4}\right)$ and of the corresponding formula for the Riemann integral of scalar functions.

\section{Weakly differentiable functions of one scalar variable.}

LEMMA 2.1. Let $\Omega$ be an open subset of $K$. If $f: \Omega \rightarrow F$ is a mapping such that for every $u \in \mathbb{F}^{\prime \prime} u \circ f$ has the $(n+1)$-th derivative at $t_{0} \in \Omega$, then $f$ has the $n$-th derivative at $t_{0}$.

Proof. Using the induction with respect to $n$, it is enough to show that the lemma is true for $n=1$.

Fix $t_{0} \in \Omega$. Without loss of generality we may assume that $t_{0}=0$. Let $\varrho>0$ be so small that $\{t:|t| \leqslant \varrho\}$ is contained in $\Omega$. Given $u \in \mathbb{F}^{\prime}$, the scalar function $u \circ f$ is of class $C^{2}$ in $\Omega$. and

Therefore there exists a function $r: \Omega \rightarrow K$ such that $\lim _{t \rightarrow 0} r(t)=0$

where

$$
u \circ f(t)=u \circ f(0)+c_{1} t+c_{2} t^{2}+r(t) t^{2}, \quad|t|<\varrho,
$$

$$
c_{1}=\left.\frac{d}{d t} u \circ f(t)\right|_{t=0}, \quad c_{2}=\left.\frac{1}{2} \frac{d^{2}}{d t^{2}} u \circ f(t)\right|_{t=0}
$$

Thus

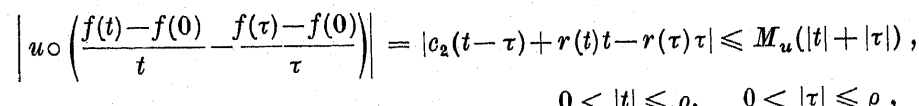

$$
0<|t| \leqslant \varrho, \quad 0<|\tau| \leqslant \varrho,
$$

where $M_{u}$ is a positive constant depending on $u \epsilon \mathbb{F}^{\prime}$ but not on $t$ nor on $\tau$. Hence for every $q \in \Gamma(F)$ there exists $M_{q}>0$ such that

$$
q\left(\frac{f(t)-f(0)}{t}-\frac{f(\tau)-f(0)}{\tau}\right) \leqslant M_{q}(|t|+|\tau|), \quad 0<|t| \leqslant \varrho, \quad 0<|\tau| \leqslant \varrho .
$$

Since $F$ is $s$-complete, this implies that there exists an element $f^{\prime}(0) \in F^{\prime}$ such that $f^{\prime}(0)=\lim _{t \rightarrow 0}(f(t)-f(0)) / t$.

3. Analytic functions from $C^{m}$ to $F$. In this section $F$ denotes a locally convex $s$-complete t.v.s. over $\boldsymbol{C}$ and $\Omega$ denotes an open subset of $\boldsymbol{C}^{n}$. If $\Omega \subset C, f: \Omega \rightarrow F$ is a continuous function and $\gamma:[a, b] \rightarrow \Omega$ is a mapping of class $C^{1}$, then the curvelinear integral of $f$ over $\gamma$ is defined by

$$
\int_{\gamma} f d z=\int_{a}^{b} f(\gamma(t)) \gamma^{\prime}(t) d t .
$$

By Section 1 the curvelinear integral always exists and it is an element of $F$. Moreover,

$$
u\left(\int_{\gamma} f d z\right)=\int_{\gamma} u \circ f d z \quad \text { for } u \in F^{\prime}
$$

THEOREM 3.1 (Characterization of analytic functions of one complex variable). Let $\Omega \subset C$. Given a mapping $f: \Omega \rightarrow F$, the following conditions are equivalent:

$\left(\mathrm{A}_{1}\right) f$ has the complex derivative $f^{\prime}$.

$\left(\mathrm{A}_{2}\right) f$ is continuous and for every compact triangle $T \subset \Omega$ we have $\int_{\partial T} f d z=0$ (if $a, b, c$ are the vertices of $T$, we put $\int_{\partial T}=\int_{[a, b]}+\int_{[b, c]}+\int_{[c, a]}$ ).

$\left(\mathrm{A}_{3}\right) f$ is continuous and for every disk $D=\left\{z \epsilon \mathrm{C}:\left|z-z_{0}\right|<r\right\}$ conained with its closure in $\Omega$ we have

$$
f(z)=(2 \pi i)^{-1} \int_{\partial D} f(t)(t-z)^{-1} d t, \quad z \in D .
$$

$\left(\mathrm{A}_{4}\right)$ At every point $a \in \Omega$ the function $f$ has all the complex derivatives $f^{(k)}(a), k=1,2, \ldots$, and

$$
f(z)=\sum_{k=0}^{\infty} \frac{1}{k !} f^{(k)}(a)(z-a)^{k}, \quad|z-a|<\varrho=\operatorname{dist}(a, \partial \Omega) .
$$

$\left(\mathrm{A}_{5}\right)$ For every point $a \epsilon \Omega$ there are elements $c_{k} \in F(k=0,1, \ldots)$ and a positive number $r$ such that

$$
f(z)=\sum_{k=0}^{\infty} c_{k}(z-a)^{k}, \quad|z-a|<r .
$$

$\left(\mathrm{A}_{6}\right)$ For every $u \in \mathbb{F}^{\prime}$ the function $u \circ f$ is analytic in $\Omega$.

Proof. $\left(\mathrm{A}_{1}\right) \Rightarrow\left(\mathrm{A}_{2}\right)$. Since for every $u \in F^{\prime}$ the function $u \circ f$ is analytic, so $0=\int_{\partial T} u \circ f d t=u\left(\int_{\partial T} f d t\right)$ for $u \in F^{\prime}$. Thus $\int_{\partial T} f d t=0$.

6 - Studia Mathematica XXXIX. 1 

Thus

$\left(\mathrm{A}_{2}\right) \Rightarrow\left(\mathrm{A}_{3}\right)$. By the Morera's theorem the function $u \circ f$ is analytic.

(*) $\quad u \circ f(z)=(2 \pi i)^{-1} \int_{\partial D} u \circ f(t)(t-z)^{-1} d t, \quad z \in D, u \in F^{\prime}$,

and this gives the result.

$\left(\mathrm{A}_{3}\right) \Rightarrow\left(\mathrm{A}_{4}\right)$. For every $u \in F^{\prime}$ the function $u \circ f$ is continuous and (*) is satisfied. So $u \circ f$ is analytic and.

$$
u \circ f(z)=\sum_{k=0}^{\infty} \frac{1}{k !}(u \circ f)^{(k)}(a)(z-a)^{k}, \quad|z-a|<\varrho, u \in F^{\prime} .
$$

By Lemma 2.1, $(u \circ f)^{(k)}=u \circ f^{(k)}, k=1,2, \ldots$ Let $0<r<\varrho$. Then by the Cauchy inequalities

$$
\left|\frac{1}{k !} u \circ f^{(k)}(a)\right| \leqslant M M_{u} r^{-k}, \quad k \geqslant 0,
$$

$M_{u}$ being a constant depending only on $u$ and $r$. Thus for every $u \epsilon F^{\prime}$ and $n \geqslant 1$

$$
\begin{aligned}
\mid u\left(f(z)-\sum_{k=0}^{n} \frac{1}{k !} f^{(k)}(a)\right. & \left.(z-a)^{k}\right)\left|\leqslant \sum_{k>n}\right| \frac{1}{k !} u \circ f^{(k)}(a)(z-a)^{k} \mid \\
& \leqslant M_{u} \frac{r}{r-|z-a|}\left(\frac{|z-a|}{r}\right)^{n+1}, \quad|z-a|<r,
\end{aligned}
$$

whence for every $q \epsilon \Gamma(F)$ and $n \geqslant 1$

$$
\begin{aligned}
q\left(f(z)-\sum_{k=0}^{n} \frac{1}{k !} f^{(k)}(a)(z-a)^{k}\right) \leqslant M_{q} \frac{r}{r-|z-a|}\left(\frac{|z-a|}{r}\right)^{n+1}, \\
|z-a|<r, n \geqslant 1 .
\end{aligned}
$$

Implications $\left(\mathbf{A}_{4}\right) \Rightarrow\left(\mathbf{A}_{5}\right),\left(\mathrm{A}_{5}\right) \Rightarrow\left(\mathrm{A}_{6}\right)$ are obvious. Implication $\left(\mathrm{A}_{6}\right)$ $\Rightarrow\left(A_{1}\right)$ follows from Lemma 2.1. The proof is concluded.

Defintion 3.1. A function $f: C \supset \Omega \rightarrow F$ is called analytic if and only if $f$ safisfies $\left(A_{5}\right)$.

CoROLLARY 3.1. If a series $\sum_{k=0}^{\infty} a_{k} z^{k}\left(a_{k} \in F^{\prime}\right)$ converges in the disk $|z|<\varrho$, then it converges uniformly on every disk $|z|<r$, where $0<|r|<\varrho$. The sum $f(z)=\sum_{k=0}^{\infty} a_{k} z^{k}$ is analytic for $|z|<\varrho$.

Corollary 3.2. The coefficients $c_{k}$ of the series in $\left(\mathrm{A}_{5}\right)$ are given by the integral formulas

$$
c_{k}=(2 \pi i)^{-1} \int_{|z-a|=r} f(z)(z-a)^{-k-1} d z, \quad k \geqslant 0 .
$$

In particular

$$
f(a)=(2 \pi)^{-1} \int_{0}^{2 \pi} f\left(a+r e^{i t}\right) d t
$$

and

$$
q\left(c_{k}\right) \leqslant \frac{M_{q}(r)}{r^{k}}, \quad k \geqslant 0, q \in \Gamma(F),
$$

where $M^{q}(r)=\sup \{q \circ f(z):|z-a|=r\}$ (Cauchy inequalities).

CoROLLARY 3.3. If $: \Omega \rightarrow F$ is analytic and $q$ is a continuous seminorm in $F$, then $q \circ f$ is a continuous subharmonic function in $\Omega$.

Indeed, it is obvious that $q \circ f$ is a real continuous function. By Corollary 3.2

$$
q \circ f(a) \leqslant(2 \pi)^{-1} \int_{0}^{2 \pi} q \circ f\left(a+r e^{i t}\right) d t
$$

so $q \circ f$ is subharmonic.

COROLLARY 3.4. If $\varphi: \Omega \rightarrow C, f: \Omega \rightarrow F$ are analytic, then $\Omega \ni z$ $\rightarrow \varphi(z) f(z) \in F$ is also analytic.

Proof. For every $u \in F^{\prime}$, the function $u_{0}(\varphi f)=\varphi u \circ f$ is analytic.

Corollary 3.5 (Three circles theorem). If $D=\left\{z \epsilon C: r_{1} \leqslant|z| \leqslant r_{2}\right\}$ is a ring contained in an open set $\Omega \subset C$, and $f: \Omega \rightarrow F$ is analytic, then for every continuous seminorm $q$ on $F$ we have

where

$$
q \circ f(z) \leqslant M_{1}^{\alpha_{12}} M_{2}^{\alpha_{21}}, \quad z \in D,
$$

$$
\alpha_{i j}=\frac{\lg \frac{|z|}{r_{j}}}{\lg \frac{r_{i}}{r_{j}}}, \quad M_{j}=\sup \left\{q \circ f(z):|z|=r_{j}\right\}(i, j=1,2) .
$$

Pro of. By Corollaries 3.3 and 3.4 the function $q\left(z^{-\lambda} f(z)\right)=|z|^{-\lambda} q \circ f(z)$ is subharmonic in $D$ for any fixed $\lambda \in \boldsymbol{R}$. By the maximum property

$$
|z|^{-\lambda} q \circ f(z) \leqslant \max \left\{M_{1} r_{1}^{-\lambda}, M_{2} r_{2}^{-\lambda}\right\}, \quad z \in D .
$$

Hence, if $\lambda$ is chosen in such a way that $M_{1} r_{1}^{-\lambda}=M_{2} r_{2}^{-\lambda}$, one gets the required inequality.

THeorem 3.2. If $\Omega \subset C^{n}$ is an open set, then for any function $f: \Omega \rightarrow F$ the following conditions are equivalent:

$\left(a_{1}\right) f$ has the partial derivatives $\frac{\partial}{\partial z_{j}} f(j=1, \ldots, n)$.

$\left(a_{2}\right)$ For every $u \in F^{\prime}$ the function $u \circ f$ is analytic. 
$\left(a_{3}\right)$ At every point $a \in \Omega$ the function $f$ has all the Gateaux differentials $\delta_{a}^{k} f, k \geqslant 1$, and

$$
f(a+x)=\sum_{k=0}^{\infty} \frac{1}{k !} \delta_{a}^{k} f(x), \quad x \in W,
$$

where $W$ is the maximal balanced neighborhood of $0 \epsilon \boldsymbol{C}^{n}$ such that $a+W \subset \Omega$ that

$\left(a_{4}\right)$ Fror every point $\alpha \epsilon \Omega$ there exists a neighborhood $W$ of $0 \epsilon C^{n}$ such

$$
f(\alpha+x)=\sum_{k=0}^{\infty} f_{k}(x), \quad x \in W
$$

where $f_{k}: C^{n} \rightarrow F$ is a homogeneous polynomial of degree $l$.

Proof. $\left(a_{1}\right) \Rightarrow\left(a_{2}\right)$. Given $u \in F^{\prime}$ the function $u \circ f$ has the partial derivatives $\frac{\partial}{\partial z_{j}} u \circ f(j=1, \ldots, n)$. So by the classical Hartogs theorem, $u \circ f$ is analytic.

$\left(a_{2}\right) \Rightarrow\left(a_{3}\right)$. By Theorem 3.1

$$
f(a+t x)=\sum_{k=0}^{\infty} f_{k}(x) t^{k}, \quad|t| \leqslant 1, x \in W
$$

where $f_{k}(x)=\left.\frac{1}{k !} \frac{d^{k}}{d t^{k}} f(a+t x)\right|_{t=0}$. In particular

$$
f(a+x)=\sum_{k=0}^{\infty} f_{k}(x), x \in W .
$$

It remains to prove that $f_{k}$ are homogeneous polynomials. But $u \circ f$ is analytic, so

$$
\left.\frac{d^{k}}{d t^{k}} u \circ f(a+t x)\right|_{t=0}=\left.\imath \circ \frac{d^{k}}{d t^{k}} f(a+t x)\right|_{t=0}
$$

is a homogeneous polynomial of degree $k$. By Corollary 4, [6],

$$
f_{k}(x)=\left.\frac{1}{l t !} \frac{d^{k}}{d t^{k}} f(a+t x)\right|_{l=0}
$$

is also a homogeneous polynomial of degree $k$.

$\left(a_{3}\right) \Rightarrow\left(a_{1}\right)$. It follows from $\left(a_{3}\right)$ that $u \circ f$ is analytic for every $u \in F^{\prime}$ Now Lemma 2.1 implies $\left(a_{1}\right)$.

One can easily check that $\left(a_{3}\right) \Rightarrow\left(a_{4}\right)$ and $\left(a_{4}\right) \Rightarrow\left(a_{2}\right)$.

Definition 3.2. A function $f: \boldsymbol{C}^{n} \supset \Omega \rightarrow F$ is called analytio if and only if $f$ satisfies $\left(a_{4}\right)$.
CoRolLARY 3.6 (Hartogs theorem). Let $E$ be a Hausdorff t.v.s. over $\boldsymbol{C}$, $\operatorname{dim} E<\infty$. Let $U$ be an open subset of $E$. If $f: U \rightarrow F$ is a function such that $f \mid V \cap U$ is analytic for every affine line $V \subset E$, then for every $x \in U$ the function $f$ has all the Gateaux differentials $\delta_{x}^{k} f, k \geqslant 1$, and

$$
f(x+h)=\sum_{k=0}^{\infty} \frac{1}{k !} \delta_{x}^{k} f(h), \quad h \in W,
$$

where $W$ is the maximal balanced neighborhood of $0 \in E$ such that $x+W \subset U$, i.e. $f$ is analytic in $U$.

COROLLARY 3.7. If $\Omega \subset C^{n}$ and $f: \Omega \rightarrow F$ is analytic, then $\partial^{a} f$ is analytic in $\Omega$ for every $a \in \boldsymbol{Z}_{+}^{n}$, where $\partial^{a}=\partial^{a_{1}+\ldots+a_{n}} / \partial z_{1}^{\alpha_{1}} \ldots \partial z_{n}^{a_{n}}$. In particular, $f$ is of class $C^{\infty}$.

Proof. It is enough to observe that by the iteration of the Cauchy integral formula we get

$$
\partial^{a} f(z)=\frac{a !}{(2 \pi i)^{n}} \int_{\partial D_{1}} \ldots \int_{\partial D_{n}} \frac{f(t)}{\left(t_{1}-z_{1}\right)^{a_{1}+1} \ldots\left(t_{n}-z_{n}\right)^{a_{n}+1}} d t_{1} \ldots d t_{n}, \quad z \in D,
$$

where $D=D_{1} \times \ldots \times D_{n}$ is any relatively compact polydisk contained in $\Omega$, and $a !=\alpha_{1} ! \ldots \alpha_{n}$ !

Proposition 3.1. Let $f_{k}: \Omega \rightarrow F, k=1,2, \ldots$, be a locally uniformly convergent sequence of analytic functions from an open set $\Omega \subset C^{n}$ to a space $F$. Then

$$
1^{\circ} f=\lim _{k \rightarrow \infty} f_{k} \text { is analytic, }
$$

$2^{\circ} \partial^{\alpha} f_{k} \rightarrow \partial^{\alpha} f$ locally uniformly in $\Omega$ for every $\alpha \in \boldsymbol{Z}_{+}^{n}$.

Proof. $1^{\circ}$ It is obvious that for every $u \in F^{\prime} \quad \lim u \circ f_{k}=u \circ f$. It follows from the locally uniform convergence of $\left\{f_{k}\right\}_{k \in N}$ that $\left\{u \circ f_{k}\right\}_{k \in N}$ is ocally bounded in $\Omega$. By the Vitali theorem the sequence $\left\{u \circ f_{k}\right\}_{k \in \boldsymbol{N}}$ converges locally uniformly and by the Weierstrass theorem, $u \circ f$ is analytic. In virtue of Theorem 3.2 the function $f$ is analytic.

$2^{\circ}$ Given $u \in F^{\prime}$ and a polydisk $D=D_{1} \times \ldots \times D_{n}$, contained in $\Omega$ with its closure, we have

$$
\partial^{\alpha}\left(u \circ f_{k}(z)\right)=\alpha !(2 \pi i)^{-n} \int_{S} \frac{u \circ f_{k}(t)}{\left(t_{1}-z_{1}\right)^{\alpha_{1}+1} \ldots\left(t_{n}-z_{n}\right)^{\alpha_{n}+1}} d t_{1} \ldots d t_{n},
$$

$u \in F^{\prime}, z \in D, k \geqslant 0$, where $S=\partial D_{1} \times \ldots \times \partial D_{n}$ and $f_{0}=f$.

Therefore

$$
\partial^{a} f_{k}(z)=\alpha !(2 \pi i)^{-n} \int_{S} \frac{f_{k}(t)}{\left(t_{1}-z_{1}\right)^{\alpha_{1}+1} \ldots\left(t_{n}-z_{n}\right)^{\alpha_{n}+1}} d t_{1} \ldots d t_{n}, \quad z \in D, k \geqslant 0,
$$


whence for every $q \in \Gamma\left(F^{\prime}\right)$ and every compact subset $\Delta \subset D$,

$$
q\left(\partial^{a} f(z)-\partial^{a} f_{k}(z)\right) \leqslant M_{q} \sup \left\{q\left(f(t)-f_{k}(t)\right): t \in S\right\}, \quad z \epsilon \Delta, k \geqslant 1,
$$

where $M_{q}$ depends on $q$ and on $\Delta$ but not on $z$ nor on $k$. The proof is completed.

Proposition 3.2 (Vitali theorem). Assume that

$1^{\circ} \Omega$ is an open connected subset of $C$,

$2^{\circ}$ a sequence of analytic functions $f_{k}: \Omega \rightarrow F^{*} k \geqslant 1$ is locally uniformly bounded in $\Omega$ and

$3^{\circ}\left\{f_{k}(x)\right\}_{k \in N}$ converges for every $x$ belonging to an open non-empty subset $U \subset \Omega$. Then $\left\{f_{k}\right\}_{k \in \mathbf{N}}$ converges locally uniformly in $\Omega$.

Proof. Fix $q \in \Gamma(F)$. By the assumption $2^{\circ}$ the sequence $\left\{q \circ f_{k}\right\}_{k \in \boldsymbol{N}}$ is locally uniformly bounded in $\Omega$. Hence, by a reasoning analogous to that in the scalar case, the Cauchy integral formula $\left(A_{3}\right)$ implies that the functions $f_{k}$ are equicontinuous with respect to the seminorm $q$ at every point $x \in \Omega$. This and the convergence of $\left\{f_{k}(x)\right\}_{k \in \boldsymbol{N}}$ for $x \in U$ imply that for every compact subset $\Delta \subset U$

$$
\sup _{x \in \Delta} q\left(f_{k}(x)-f_{l}(x)\right)=\varepsilon_{k, l} \rightarrow 0, \quad \text { as } k, l \rightarrow \infty .
$$

The proof will be completed if we show that $(*)$ holds true for every compact subset $\Delta \subset \Omega$. Because then, $F$ being $s$-complete, the sequence $\left\{f_{k}(x)\right\}_{k \in \boldsymbol{N}}$ has a limit $f(x)$ for each $x \epsilon \Omega$, and again by $(*)$ the convergence is locally uniform in $\Omega$.

Thus it is enough to show that the set

$$
\Omega_{0}=\{a \epsilon \Omega: \text { there exists a disk } D \text { with center } a \text { such that }
$$

$$
\left.\sup _{x \in D} q\left(f_{k}(x)-f_{l}(x)\right) \rightarrow 0 \text { as } k, l \rightarrow \infty\right\}
$$

is identical with $\Omega$. In order to prove that $\Omega_{0}=\Omega$, it is enough to show that if $a \in \Omega_{0}$ and $D$ is any disk with center $a$ and radius $r$ such that the closure $\bar{D} \subset \Omega$, then $D \subset \Omega_{0}$. Let $G=\left\{x \in C:|x-a| \leqslant r_{2}\right\}$ be a closed disk such that $G \subset \Omega$ and $r<r_{2}$. Let $r_{1}>0$ be so small that $\{x \in C:|x-a|$ $\left.<r_{1}\right\} \subset \Omega_{0}$ and $r_{1}<r$. It follows from Corollary 3.5 that

$$
q\left(f_{k}(x)-f_{l}(x)\right) \leqslant \varepsilon_{k, l}^{\beta(x)}(M+1), \quad x \in G, \quad k, l \geqslant 1,
$$

where $\quad \varepsilon_{k, l}=\sup _{|x-a| \leqslant r_{1}} q\left(f_{k}(x)-f_{l}(x)\right), \quad M=\sup _{k, l \geqslant 1} \sup _{x \in G} q\left(f_{k}(x)-f_{l}(x)\right)$ and $\beta(x)=\frac{\lg \frac{|x-a|}{r_{2}}}{\lg \frac{r_{1}}{r_{2}}}$.
According to $(*), \varepsilon_{k l} \rightarrow 0$ as $k, l \rightarrow \infty$.

Consequently

$$
\sup _{x \in D} q\left(f_{k}(x)-f_{l}(x)\right) \leqslant(M+1) \varepsilon_{k l}^{a} \rightarrow 0 \quad \text { as } l, l \rightarrow \infty,
$$

where $\alpha=\frac{\lg \frac{r}{r_{2}}}{\lg \frac{r_{1}}{r_{2}}}$.

Thus $D \subset \Omega_{0}$. The proof is completed.

4. A polynomial lemma. Given a compact set $X \subset \boldsymbol{C}$, we put (4.1) $L(z, X)=\sup _{k \geqslant 1}\left\{\sup \left\{|f(z)|^{1 / k}: f\right.\right.$ is a polynomial of one complex variable such that $\operatorname{deg} f \leqslant k$ and $\|f\| \leqslant 1\}\}, \quad z \epsilon \boldsymbol{C}$,

where $\|f\|=\sup \{|f(x)|: x \in X\}$. It is known [21] that if the transfinite diameter (logaritmic capacity) $d(X)>0$, then

$$
L(z, X)=\left\{\begin{array}{cl}
1, & \text { if } z \epsilon \boldsymbol{C} \backslash D_{\infty}, \\
\exp G(z), & \text { if } z \in D_{\infty},
\end{array}\right.
$$

where $D_{\infty}$ is the unbounded component of $C \backslash X$ and $G$ is the Green's function of $D_{\infty}$ with pole at $\infty$.

We shall need the following

LEMMA 4.1. Let $X$ be a compact subset of $\boldsymbol{C}$ such that the unbounded component of $C \backslash X$ is regular with respect to the classical Dirichlet problem. Let $\left\{X_{s}\right\}_{s \in \boldsymbol{N}}$ be a sequence of compact subsets of $X$ such that $X_{s} \subset X_{s+1}, \bigcup_{s=1}^{\infty} X_{s}$ $=X . P u t$

Then

$$
L^{*}\left(x, X_{s}\right)=\limsup _{y \rightarrow x} L\left(y, X_{s}\right), \quad x \in C .
$$

$$
L(x, X)=\lim _{s \rightarrow \infty} L^{*}\left(x, X_{s}\right), \quad x \in \boldsymbol{C},
$$

the convergence being uniform on every compact subset of $\boldsymbol{C}$.

Proof. By Lemma 4.2 and 4.4 of [21] the sequence $\left\{L^{*}\left(x, X_{s}\right)\right\}_{s \in \mathbf{N}}$ is decreasing and,

$$
L(x, X)=\lim _{s \rightarrow \infty} L^{*}\left(x, X_{s}\right), \quad x \in \boldsymbol{C} .
$$

The limit function being continuous, we conclude that the convergence is locally uniform in $\boldsymbol{C}$.

Remark 4.1. It is well known that if $X \subset C$ is a continuum (compact, connected set containg more than one point), then $D_{\infty}$ is regular with respect to the Dirichlet problem. 
Polinnomal Lemma. Let $\Phi=\left\{f: C^{n} \rightarrow F\right\}$ be a family of polynomials of $n$ complex variables with values in a locally convex space $F$ over $C$. Let $X_{j}(j=1, \ldots, n)$ be a compact subset of $C$ such that the unbounded component of $\boldsymbol{C} \backslash X_{j}$ is regular with respect to the Dirichlet problem. If

$$
\sup _{f \in \Phi} q \circ f(z)<\infty, \quad z \in X=X_{1} \times \ldots \times X_{n}, q \in \Gamma(F),
$$

then for every $\omega>1$ there exists $\delta>0$ such that for every $q \in \Gamma(F)$ one can find $M_{q}>0$ such that

$$
q \circ f(z) \leqslant M_{q} \omega^{\operatorname{deg} f}, \quad z \in X_{1}^{(\delta)} \times \ldots \times X_{n}^{(\delta)}, \quad f \epsilon \Phi,
$$

where $X_{j}^{(\delta)}=\left\{z \epsilon C\right.$ : dist $\left.\left(z, X_{j}\right)<\delta\right\}(j=1, \ldots n)$.

Proof. $1^{\circ} n=1$. Given $\omega>1$, put $\omega_{1}=(1+\omega) / 2$. Let $k_{0}$ be so large that $\sqrt[k]{k+1} \omega_{1}<\omega$ for $k>k_{0}$, and let $\delta>0$ be so small that

$$
L(z, X)<\omega_{1}, \quad \text { if } \operatorname{dist}(z, X) \leqslant \delta
$$

Given $q \in \Gamma(F)$, put

$$
X_{j}=\left\{x \in X: \sup _{f \in \Phi} q \circ f(x) \leqslant j\right\}, \quad j=1,2, \ldots
$$

The sets $X_{j}$ are compact, $X_{j} \subset X_{j+1}$ and $\bigcup_{j=1}^{\infty} X_{j}=X$. Therefore by Lemma 4.1,

$$
\lim _{j \rightarrow \infty} L^{*}\left(z, X_{j}\right)=L(z, X), \quad z \epsilon \boldsymbol{C}
$$

the convergence being locally uniform. So we may take $j$ so large, that

$$
L^{*}\left(z, X_{j}\right)<\omega_{1}, \quad \text { if } \operatorname{dist}(z, X)<\delta .
$$

Let $j$ be any arbitrary fixed integer such that $(*)$ holds true. Let $\xi^{(k)}=\left\{\xi_{k 0}, \ldots, \xi_{k k}\right\}, k=1,2, \ldots$, denote the $k$-th system of Felkete points of $X_{j}[10]$. It is well known that the fundamental polynomials of Lagrange, corresponding to the system of nodes $\xi^{(k)}$, satisfy the following inequalities

$$
\left|L^{(s)}\left(z, \xi^{(k)}\right)\right| \leqslant 1, \quad z \epsilon X_{j}, s=0, \ldots, k .
$$

Therefore by (4.1)

$$
\left|L^{(s)}\left(z, \xi^{(k)}\right)\right| \leqslant\left(L^{*}\left(z, X_{j}\right)\right)^{k}, \quad z \in C, s=0, \ldots, k .
$$

If $f \in \Phi$ and $\operatorname{deg} f=k$, then by the Lagrange interpolation formula

whence

$$
f(z)=\sum_{s=0}^{k} L^{(s)}\left(z, \xi^{(k)}\right) f\left(\xi_{k, s}\right), \quad z \epsilon \boldsymbol{C},
$$

$$
q \circ f(z) \leqslant(k+1) j\left(L^{*}\left(z, X_{j}\right)\right)^{k}, \quad z \epsilon \boldsymbol{C}
$$

Consequently, if $k>k_{0}$ we have

$q \circ f(z) \leqslant j \omega^{k}, \quad \operatorname{dist}(z, X)<\delta, \quad f \epsilon \Phi, \quad \operatorname{deg} f=k, \quad q \in \Gamma(F)$,

the number $j$ depending only on $q$ but not on $f$, nor on $\delta$, nor on $z$.

If $k \leqslant k_{0}$, then for $f \epsilon \Phi, \operatorname{deg} f=k, q \epsilon \Gamma(F)$ we have

$$
q \circ f(z) \leqslant\left(\sum_{s=0}^{k_{0}} q \circ f\left(\xi_{k_{0} s}\right)\right)\left(L^{*}\left(z, X_{j}\right)\right)^{k_{0}} \leqslant H_{q} \omega^{k}, \quad \operatorname{dist}(z, X)<\delta,
$$

where $H_{q}$ is suitably chosen. To end the proof, it is enough to define $M_{q}=\max \left\{j, H_{q}\right\}$.

$2^{\circ} n \geqslant 2$. This case may be easily obtained by the induction with respect to $n$.

Proposition 4.1. Let $U$ be an open subset of $K^{n}$. If the series $\sum_{k=0}^{\infty} f_{k}$ of homogeneous polynomials $f_{k} \epsilon Q^{k}\left(K^{n} F\right)$ is convergent in $U$, then for every compact subset $G \subset U$ there exists $\theta \in(0,1)$ such that

$$
q \circ f_{k}(z) \leqslant M_{q} \theta^{k}, \quad z \epsilon G, k \geqslant 0, q \epsilon \Gamma\left(F^{\prime}\right),
$$

$M_{q}$ being a constant depending only on $q$ and $G$.

Proof. Given $x_{0} \in U$, let $X=X_{1} \times \ldots \times X_{n}$ be a compact subset of $U$ such that $X_{j} \subset K$ is connected and $x_{0} \in$ int $X$. By the Polynomial Lemma for every $\omega>1$ and for every $q \in \Gamma(F)$ there exists $M_{q}$ such that

Hence

$$
q \circ f_{k}(z) \leqslant M_{q} \omega^{k}, \quad z \epsilon X, k \geqslant 0 .
$$

$$
q \circ f_{k}\left(\frac{\theta}{\omega} z\right) \leqslant M_{q} \theta^{k}, \quad z \in X, k \geqslant 0, \theta>0,
$$

$$
q \circ f_{k}(z) \leqslant M_{q} \theta^{k}, \quad z \epsilon \frac{\theta}{\omega} X, k \geqslant 0
$$

If $\frac{\theta}{\omega}$ is sufficiently close to 1 , then $x_{0} \in$ int $\frac{\theta}{\omega} X$. Therefore by the Borel-Lebesque theorem, the proposition is proved.

Remark 4.2. Polynomial Lemma and the Proposition 4.1 remain true if one replaces formally $q \epsilon \Gamma(F)$ by $u \in F^{\prime}$. In the proof it suffices to replace $q$ by $|u|$.

5. Series of homogeneous polynomials. Let $E, E_{1}, F$ be t.v.s. over $K$ an let $U$ denote an open subset of $E$. We always assume that $F$ is locally convex and $s$-complete. 
DEFINITION 5.1. A series $\sum_{n=0}^{\infty} f_{n}$ of homogeneous polynomials $f_{n} \in Q^{n}\left(E, E_{1}\right)$ will shortly be called a formal series from $E$ to $E_{1}$. The space of all formal series with continuous terms will be denoted by $S\left(E, E_{1}\right)$.

DEFINITION 5.2. We say that a formal series $\sum_{n=0}^{\infty} f_{n}$ converges in a set $A \subset E$ if for every $x \in A$ the series $\sum_{n=0}^{\infty} f_{n}(x)$ is convergent.

DEFINITION 5.3. We say that a formal series $\sum_{n=0}^{\infty} f_{n} \in S(E, F)$ converges normally at zero (at a point $a \in E$ ), if for every $q \in \Gamma(F)$ there exists a neighborhood $W_{q}$ of $0 \in E$ (of the point $a$ ) such that

$$
\sum_{n=0}^{\infty} \sup \left\{q \circ f_{n}(x): x \in W_{q}\right\}<\infty .
$$

Definition 5.4. Given a formal series $\sum_{n=0}^{\infty} f_{n}$, the interior $H$ of the set $\left\{x \in E: \sum_{n=0}^{\infty} f_{n}(x)\right.$ is convergent $\}$ will be called a domain of convergence of the series $\sum_{n=0}^{\infty} f_{n}$.

DeFinition 5.5. A function $f: U \rightarrow E_{1}$ is called $G$-analytic in $U$, if for every $x \in U$ there exists a formal series $\sum_{n=0}^{\infty} f_{n}$ from $E$ to $E_{1}$ such that

$$
f(x+h)=\sum_{n=0}^{\infty} f_{n}(h)
$$

for all $h$ in a neighborhood of $0 \in E$.

DeFInITION 5.6. A continuous function $f: U \rightarrow E_{1}$ is called analytic in $U$, if for every point $x \in U$ there exists a series $\sum_{n=0}^{\infty} f_{n} \in S\left(E, E_{1}\right)$ such that

$$
f(x+h)=\sum_{n=0}^{\infty} f_{n}(h)
$$

for all $h$ in a neighborhood of $0 \in E$.

Defintrion 5.7. A function $f: U \rightarrow E_{1}$ is called weakly-analytic in $U$, if for every $u \in E_{1}^{\prime}$ the function $u \circ f$ is analytic.

It is obvious that $G$-analyticity and weak-analyticity are implied by the analyticity.

Propostiton 5.1. If a formal series $\sum_{n=0}^{\infty} f_{n} \in S(E, F)$ converges in a neighborhood of $0 \in E$ to a limit $f$ which is continuous at 0 , then the series converges normally at zero.
Proof. Given $q \in \Gamma(F)$, let $W$ be a balanced neighborhood of zero such that $q(f(h)) \leqslant M_{q}<\infty$ for $h \epsilon W$ and $f(h)=\sum_{n=0}^{\infty} f_{n}(h), h \epsilon W$. Then $f(t h)=\sum_{n=0}^{\infty} f_{n}(h) t^{n},|t| \leqslant 1$. Therefore by the Cauchy inequalities,

$$
q \circ f_{n}(h) \leqslant M_{q}, \quad h \in W, n \geqslant 0 .
$$

Thus $q \circ f_{n}(h) \leqslant M_{q} 2^{-n}, h \in W_{q}=\frac{1}{2} W$. The proof is concluded.

PRoposition 5.2. Let $E$ be a Baire space.

$1^{\circ}$ If a series $\sum_{n=0}^{\infty} f_{n} \in S(E, F)$ converges in an absorbing subset of $E$, then it converges normally at zero.

$2^{\circ}$ If a series $\sum_{n=0}^{\infty} f_{n} \in S(E, F)$ converges in a neighborhood $U$ of $0 \in E$, then $f=\sum_{n=0}^{\infty} f_{n}$ is continuous at 0 .

Proof. $1^{\circ}$. Take a fixed $q \epsilon \Gamma(F)$. It follows from the assumptions that $q \circ f_{n}(x) \leqslant 2^{-n}, n=0,1, \ldots$, for all $x$ belonging to an absorbing subset $A$ of $E$. The functions $f_{n}$ being continuous, the last inequalities are still valid in the closure $\bar{A}$. Since $E$ is a Baire space, so int $\bar{A} \neq \emptyset$. Therefore there exists a point $a \epsilon E$ and a balanced neighborhood $W$ of $0 \epsilon E$, such that $q \circ f_{n}(x) \leqslant 2^{-n}(n \geqslant 0)$ for $x \in a+W$. By Lemma $2[6], q \circ f_{n}(x) \leqslant 2^{-n}$, $x \epsilon \frac{1}{2 e} W$.

$2^{\circ}$ Fix $\varepsilon>0$ and $q \epsilon \Gamma(F)$. According to $1^{\circ}$ the series $\sum_{n=0}^{\infty} f_{n}$ is normally convergent at zero, so $\sum_{n>k} q \circ f_{n}(x)<\varepsilon / 2$ for all $x$ in a neighborhood $W_{1}$ of zero in $E, k$ being a positive integer sufficiently large. Observe that

$$
q(f(x)-f(0)) \leqslant \sum_{n=0}^{k} q \circ f_{n}(x)+\sum_{n>k} q \circ f_{n}(x), \quad x \in W_{1} .
$$

Since $f_{n}$ are continuous, there exists a neighborhood $W_{2}$ of $0 \epsilon E$ such that the first sum is uniformly bounded by $\varepsilon / 2$ in $W_{2}$. Therefore $q(f(x)-$ $-f(0))<\varepsilon$ for $x \in W_{1} \cap W_{2}$. So $f$ is continuous at 0 .

Proposition 5.3. The domain of convergence $H$ of any format series $\sum_{n=0}^{\infty} f_{n}$ from $E$ to $F$ is either empty or a balanced neighborhood of $0 \in E$. For every point $x \in H$ and for each $q \in \Gamma(F), \sum_{n=0}^{\infty} q \circ f_{n}(x)<\infty$.

Proof. At first we shall prove that if $a \in H$ and $W$ is a balanced neighborhood of $0 \in E$ such that $a+W \subset H$, then $\frac{1}{2 e} W \subset H$. To this aim let $x$ be a fixed point of $W$. Then $\sum_{n=0}^{\infty} f_{n}(\tau a+t x)$ is a series of homogeneous 
polynomials of $(\tau, t) \epsilon K^{2}$ of respective degrees $n$, which converges for $\tau$ in a neighborhood of 1 and $t \epsilon(-1-\delta, 1+\delta)$, where $\delta>0$ is sufficiently small. By Proposition 4.1 there exist $\theta \in(0,1)$ and $M_{q}>0$ such that

$$
q \circ f_{n}(a+t x) \leqslant M_{q} \theta^{n}, t \in[-1,1], q \in \Gamma(F) .
$$

This implies, by Lemma 2 [6], that

$$
q \circ f_{n}(t x) \leqslant M_{q} \theta^{n}, \quad t \epsilon\left[-\frac{1}{2 e}, \frac{1}{2 e}\right], q \epsilon \Gamma(F), n \geqslant 0 .
$$

Since $F$ is $s$-complete, this implies that $\sum_{n=0}^{\infty} f_{n}$ converges in $\frac{1}{2 e} W$.

Thus, if $H \neq \varnothing, H$ is a neighborhood of $0 \in E$ and $\sum_{n=0}^{\infty} q \circ f_{n}(x)<\infty$ for $x \in H, q \in \Gamma(F)$.

It remains to prove that $H$ is balanced, i.e. $t H \subset H$ for $t \in K,|t| \leqslant 1$.

Put $y_{0}=t_{0} x_{0}$, where $\left|t_{0}\right| \leqslant 1$ and $x_{0} \in H$. We already know that $y_{0} \in H$, if $y_{0}=0$. If $y_{0} \neq 0$, then $t_{0} \neq 0$ and the mapping $\Phi: E \ni x \rightarrow t_{0} x \in E$ is a homeomorphism. So $y_{0}$ is an interior point of $\Phi(H)$. Since the series $\sum_{n=0}^{\infty} f_{n}(y)$ is, of course, convergent at every point $y \in \Phi(H)$, it follows that $y_{0} \in H$. Thus $H$ is an open balanced neighborhood of $0 \epsilon E$.

Proposition 5.4. Assume that $K=\boldsymbol{R}$. If a formal series $\sum_{n=0}^{\infty} f_{n}$ from $E$ to $F$, converges in an open set $H \subset E$ (or the sequence $\left\{f_{n}(x)\right\}_{n \in \boldsymbol{N}}$ is bounded at every point $x \in H)$, then there exists an open set $\tilde{H} \subset \tilde{E}$ such that $H \subset \tilde{H}$ and the complexified series $\sum_{n=0}^{\infty} \tilde{f}_{n}$ is convergent in $\tilde{H}$.

Proof. Given $a \in H$, let $U$ be a balanced neighborhood of $0 \epsilon E$ such that $a+x+t y \in H$, when $x, y \in U,-1 \leqslant t \leqslant 1$. Since $\sum_{n=0}^{\infty} f_{n}$ is convergent
at every point $x \in H$, we have

$$
\sup _{n \geqslant 0} q \circ f_{n}(a+x+t y)<\infty, \quad t \epsilon[-1,1], q \in \Gamma(F), x, y \in U .
$$

So we may apply the Polynomial Lemma to the family

$$
\Phi=\left\{\boldsymbol{C} \ni t \rightarrow \tilde{f}_{n}(a+x+t y) \epsilon \tilde{F}\right\}_{n \in \mathbf{N}},
$$

if $x, y$ are any fixed points of $U$.

Let $\omega>1$ be so close to 1 , that $\left(\omega^{2}-1\right) a \in U$. By the Polynomial Lemma we may find $\delta>0$ such that for every $q \in \Gamma(F)$, and $x, y \in U$, there exists $M=M(q, x, y)$ such that

$\tilde{q} \circ \tilde{f}_{n}(a+x+(t+i \tau) y) \leqslant M \omega^{n}, \quad n \geqslant 1,-\delta \leqslant \tau \leqslant \delta,-1 \leqslant t \leqslant 1$.

Hence, deviding both sides by $\omega^{2 n}$ and putting $t=0, \tau=\delta$, we get

$$
\tilde{q} \circ \tilde{f}_{n}\left(\omega^{-2}(a+x)+i \delta \omega^{-2} y\right) \leqslant M \omega^{-n}, \quad n \geqslant 1, x, y \in U .
$$

The mapping $T: E \ni x \rightarrow \omega^{-2} \quad(a+x) \in E$ is a homeomorphism, so $V=T(U)$ is an open set. Since $\left(\omega^{2}-1\right) a \in U$, so $T\left(\left(\omega^{2}-1\right) a\right)=a \epsilon \nabla$. Let $W$ be a balanced neighborhood of $0 \epsilon E$ such that $a+W \subset V$ and $W$ $\subset \delta \omega^{-2} U$. It follows from inequality $(*)$ that

$$
\tilde{q} \circ \tilde{f}_{n}(a+x+i y) \leqslant M \omega^{-n}, \quad x, y \in W, n \geqslant 1 .
$$

Therefore for every $a \in H$ we can find a balanced neighborhood $W_{a}$ of $0 \in E$ such that the series $\sum_{n=0}^{\infty} \tilde{f}_{n}$ converges at every point $x \in a+W_{a}+i W_{a}$. The open set $\tilde{H}$, given by $\tilde{H}=\bigcup_{a \in H}\left(a+W_{a}+i W_{a}\right)$, satisfies the required properties.

PropostTION 5.5. If $K=C$, then a function $f: U \rightarrow F$ is $G$-analytic if and only if for every affine line $V \subset \mathbb{E}$ the function $f \mid U \cap V$ is analytic. Moreover, if $f$ is $G$-analytic, then $f_{\epsilon} G^{\infty}(U)$ and

$$
f(x+h)=\sum_{n=0}^{\infty} \frac{1}{n !} \delta_{x}^{n} f(h) \quad \text { for } h \in W,
$$

where $W$ is the maximal balanced neighborhood of $0 \epsilon E$ such that $x+W \subset U$.

Proof. Necessity. The function $f \mid U \cap V$ is analytic for every affine line $V \subset E$, because

$$
f(x+t h)=\sum_{n=0}^{\infty} f_{n}(h) t^{n}, \quad|t| \leqslant 1, h \in W .
$$

Sufficiency. Assume that $f \mid U \cap V$ is analytic for every affine line $\nabla \subset E$, and let $W$ be the maximal balanced neighborhood of $0 \epsilon E$ such that $x+W \subset U$. By Corollary 3.6 $f \mid U \cap V$ is analytic for every affine subspace $V \subset E, \operatorname{dim} V<\infty$. Thus, by Theorem 4 [6], the function $f_{n}$ given by

$$
f_{n}(h)=\frac{1}{n !} \delta_{x}^{n} f^{\prime}(h)=\left.\frac{1}{n !} \frac{d^{n}}{d t^{n}} f(x+t h)\right|_{t=0}, \quad h \in E,
$$

is a homogeneous polynomial of degree $n$. Hence, by Theorem 3.1, we have $\dot{f}(x+h)=\sum_{n=0}^{\infty} f_{n}(h), h \in W$.

THeOREM 5.1. If a formal series $\sum_{n=0}^{\infty} f_{n}$ from $E$ to $F$ converges in an open set $H \subset E$, then $f=\sum_{n=0}^{\infty} f_{n}$ is G-analytic in $H$.

Proof. (a) $K=\boldsymbol{C}$. By Proposition 4.1 the series $\sum_{n=0}^{\infty} f_{n}$ converges uniformly on every compact subset of $H \cap V$, where $V$ is any vector subspace 
of $E, \operatorname{dim} V=2$. Thus for each affine line $V \subset E$ the function $f \mid H \cap V$ is analytic. Therefore by Proposition 5.5, $f$ is $G$-analytic.

(b) $K=\boldsymbol{R}$. By Proposition 5.4 the complexified series $\sum_{n=0}^{\infty} \tilde{f}_{n}$ converges in an open subset $\tilde{H} \subset \tilde{E}, H \subset \tilde{H}$. By (a) its sum $\tilde{f}$ is $G$-analytic in $\tilde{H}$. Hence $f=\tilde{f} \mid H$ is $G$-analytic in $H$.

COROLLARY 5.1. A function $f: U \rightarrow F$ is G-analytic if and only if $f \in G^{\infty}(U)$ and for every $x \in U$ there is a neighborhood $W$ of $0 \epsilon E$ such that

$$
f(x+h)=\sum_{n=0}^{\infty} \frac{1}{n !} \delta_{x}^{n} f(h), \quad h \in W .
$$

THeOREM 5.2. If $E$ is a Baire space and if a formal series $\sum_{n=0}^{\infty} f_{n}$ with continuous terms converges in an open set $H \subset E$, then $f=\sum_{n=0}^{\infty} f_{n}$ is analytic in $H$.

Proof. By Theorem 5.1 the function $f$ is $G$-analytic. So, by Proposition 5.2, it is enough to show that the Gateaux differentials $\delta_{x}^{n} f, n \geqslant 1$, are continuous for any fixed $x \in H$.

(a) $K=C$. By Proposition 4.1 the series $\sum_{n=0}^{\infty} f_{n}$ converges uniformly on every compact subset of $H \cap V$, where $V$ is any 2-dimensional vector subspace of $E$. Therefore, given $x \in H$ and $h \epsilon E$, the series $\sum_{n=0}^{\infty} f_{n}(x+t h)$ converges uniformly with respect to $t$ belonging to a neighborhood of $0 \in \boldsymbol{C}$. Thus, by Proposition 3.1, we have

$$
\left.\frac{d^{k}}{d t^{k}} f(x+t h)\right|_{t=0}=\left.\sum_{n=0}^{\infty} \frac{d^{k}}{d t^{k}} f_{n}(x+t h)\right|_{t=0}, \quad h \in E, k \geqslant 1 .
$$

This may be written in the following equivalent form

But

$$
\delta_{x}^{k} f(h)=\sum_{n=0}^{\infty} \delta_{x}^{k} f_{n}(h), \quad h \in E, k \geqslant 1 .
$$

$$
\delta_{x}^{k} f_{n}(h)=k !\left(\begin{array}{l}
n \\
k
\end{array}\right) \bar{f}_{n}(\underbrace{x, \ldots, x}_{n-k}, \underbrace{h, \ldots, h}_{k})
$$

is a continuous polynomial of degree $k$. So by Theorem $2,[6]$, the polynomial $\delta_{x}^{k} f$ is also continuous.

(b) $K=\boldsymbol{R}$. By Theorem 3, [6], the polynomials $\tilde{f}_{n}$ are continuous and by the Proposition 5.4 the complexified series $\sum_{n=0}^{\infty} \tilde{f}_{n}$ converges in an open subset $\tilde{H} \subset \tilde{E}, H \subset \tilde{H}$. By (a)

$$
\delta_{x}^{k} \tilde{f}(h)=\sum_{n=0}^{\infty} \delta_{x}^{k} \tilde{f}_{n}(h), \quad x \in \tilde{H}, h \in \tilde{E}, k \geqslant 1
$$

where $\tilde{f}=\sum_{n=0}^{\infty} \tilde{f}_{n}$. In particular,

$$
\delta_{x}^{k} f(h)=\sum_{n=0}^{\infty} \delta_{x}^{k} f_{n}(h), \quad x \in H, h \in E, k \geqslant 1 .
$$

By Theorem 2, [6], the polynomial $\delta_{x}^{k} f$ is also continuous.

Corollary 5.2 (Riemann theorem on removable singularities). Let $A$ be a relatively closed subset of $U \subset E(E$ is a Baire t.v.s. over $C)$ and let $f: U \backslash A \rightarrow F$ be an analytic function such that for every affine line $V \subset E$ the set $A \cap V$ is an isolated subset of the topological space $U \cap V$ and $f \mid(U \backslash A) \cap V$ is bounded in a neighborhood of any point of $A$. Then there exists a unique analytic function $f^{*}: U \rightarrow F$ such that $f^{*} \mid U \backslash A=f$.

Proof. It is obvious that $U \backslash A$ is nowhere dense. One may easily check that $U=\bigcup_{x \in U \backslash A}\left(x+W_{x}\right)$, where $W_{x}$ is the maximal balanced neighborhood of $0 \in \mathbb{E}$ such that $x+W_{x} \subset U$. Given $x \in U \backslash A$, we have

$$
f(x+h)=\sum_{n=0}^{\infty} f_{n}(h), \quad h \in V_{x},
$$

where $V_{x}$ is a balanced neighborhood of $0 \epsilon E$ such that

$$
x+V_{x} \subset U \backslash A, \quad \text { and } \quad f_{n}=\frac{1}{n !} \delta_{x}^{n} f .
$$

By the classical Riemann theorem the series $\sum_{n=0}^{\infty} f_{n}(h)$ converges for $h \epsilon W_{x}$. By Theorem 5.2 the function $f_{x}(h)=\sum_{n=0}^{\infty} f_{n}(x-h), h \in x+W_{x}$, is analytic. It is clear that $f^{*}=\bigcup_{x \in U \backslash A} f_{x}$ gives the required continuation of $f$.

Countrexample 5.1. Let $E$ be a normed space over $C$ composed of all the points $x=\left(x_{1}, x_{2}, \ldots\right) \epsilon t^{2}$ which have only finitely many coordinates different from 0 . The function $f: E \rightarrow C$, defined by the series with continuous terms $f(x)=\sum_{n=0}^{\infty}\left(n x_{n}\right)^{n}, x \in E$, is $G$-analytic but it is not analytic, because $f$ is not continuous.

This example shows that Theorem 5.2 breaks off to be true if $E$ is not a Baire space.

DEFINITION 5.8. We say that $f: U \rightarrow F$ is continuous at $a \in U$ with respect to a seminorm $q$ (or shortly that $f$ is $q$-continuous at a) if for every $\varepsilon>0$ there exists a neighborhood $V$ of a such that $q(f(x)-f(a))<\varepsilon$ for $x \in V$.

It is obvious that $f$ is continuous if and only if it is $q$-continuous for every $q \epsilon \Gamma(F)$. 
An inspection of the proof of Theorem 5.2 permits to formulate tho following

Remark 5.1. If $E$ is a Baire space, $f_{n} \epsilon Q^{n}(E, F)$ are $q$-continuous and $H$ is the domain of convergence of $\sum_{n=0}^{\infty} f_{n}$, then $f=\sum_{n=0}^{\infty} f_{n}$ is $q$-continuous in $H$ and for every $x \in H$ all the Gateaux differentials $\delta_{x}^{n} f(n \geqslant 1)$ are $q$-continuous.

Propositron 5.6. A formal series $\sum_{n=0}^{\infty} f_{n}$ from $E$ to $F$ converges in an open set $U$ if and only if $\sum_{n=0}^{\infty} f_{n}$ weakly converges in $U$.

Proof. Let $x$ be a fixed point of $U$. Let $\omega>1$ be so close to 1 that $\omega x \in U$. Then

whence

and finally

$$
\left|u \circ f_{n}(\omega x)\right| \leqslant M_{u}, \quad u \in F^{\prime}, n \geqslant 0
$$

$$
q \circ f_{n}(\omega x) \leqslant M_{q}, \quad q \in \Gamma(F), n \geqslant 0,
$$

$$
q \circ f_{n}(x) \leqslant M_{q} \omega^{-n}, \quad q \in \Gamma(F), n \geqslant 0 .
$$

6. Analytic functions in topological vector spaces over $C$. In this section all t.v.s. are over $\boldsymbol{C}$ (except Lemma 6.1 and Proposition 6.6); $U$ is an open subset of t.v.s. $E$.

Lemira 6.1. Let $U$ and $U_{0}$ be open subsets of a t.v.s. $E$ over $K$ such that $U$ is connected, $U_{0} \neq \varnothing$ and $U_{0} \subset U$. Consider the following condition:

(C) If $x \in U_{0}$ and $W$ is a balanced neighborhood of $0 \in E$ such that $x+W \subset U$, then $x+W \subset U_{0}$.

If (C) is satisfied, then $U_{0}=U$.

Pro of. Suppose $U \cap \partial U_{0} \neq \varnothing$ and let $b \epsilon U \cap \partial U_{0}$. Choose a balanced neighborhood $W$ of zero in $E$ such that $b+W+W \subset U$ and let $a \epsilon(b+W)$ $\subset U_{0}$. We claim that $a+W \subset U$. Indeed, any $x \in a+W$ may be written in the form $x=a+w, w \in W$. Since $a=b+w^{\prime}, w^{\prime} \in W$, so $x=b+w^{\prime}+$ $+w \in b+W+W \subset U$. Thus $a+w \in U$. It follows from (C) that $a+W \subset U_{0}$. The equation $b=a-w^{\prime}\left(w^{\prime} \in W\right.$ ) implies that $b \in a+W$. Thus $b \in U_{0}$. This contradition concludes the proof.

Proposimion 6.1. Let $q$ be a continuous seminorm in $F$, let $U$ be a neighborhood of a point $a \in E$ and let $\mathscr{A}$ be a familly of $G$-analytic functions $f: U \rightarrow F$ such that

$$
\sup \{q \circ f(x): f \in \mathscr{A}, x \in U\} \leqslant M<\infty .
$$

Then all the functions $f \in \mathscr{A}$ are $q$-equicontinuous at a, i.e. for every $\varepsilon>0$ there exists a balanced neighborhood $W$ of $0 \in E$ such that $a+W \subset U$ and

$$
q(f(a+x)-f(a))<\varepsilon, \quad x \in W, f \in \mathscr{A}
$$

Pro of. By $G$-analyticity $f(a+x)=\sum_{n=0}^{\infty} f_{n}(x), x \in W$, where

$$
f_{n}(x)=\frac{1}{n !} \delta_{a}^{n} f(x)=(2 \pi i)^{-1} \int_{|t|=1} f(a+t x) t^{-n-1} d t \quad \text { for } x \in W .
$$

So $q \circ f_{n}(x) \leqslant M, x \in W, n \geqslant 0, f \in \mathscr{A}$. Therefore

$$
q(f(a+x)-f(a)) \leqslant \sum_{n=1}^{\infty} q \circ f_{n}(x) \leqslant M \frac{\theta}{1-\theta}, \quad x \in \theta W, 0<\theta<1, f \in \mathscr{A} .
$$

This proof and Theorem 1, [6], imply the following

CoROLlaRy 6.1. If $U$ is a neighborhood of $a \in E$ and $f: U \rightarrow F$ is $a G$ analytic function such that the function $q \circ f$ is bounded in $U$, then $f$ is $q$-continuous at $a$, and the Gateaux differentials $\delta_{a}^{n} f(n \geqslant 1)$ are $q$-continuous in $E$.

Given a function $f: U \rightarrow F$, consider the following conditions:

(a) $\forall q \in \Gamma(F)$ the function $q \circ f$ is locally bounded in $U$.

(b) $\forall q \in \Gamma(F)$ there exists an open non-empty subset $W_{q} \subset U$ such that $q \circ f$ is bounded in $W_{q}$.

(c) $f$ is continuous at a point $x_{0} \in U$.

(d) $f$ is continuous in $U$.

(e) $f$ is analytic in $U$.

TheOREM 6.1. Let $f: U \rightarrow F$ be a G-analytic function. Then

(i) For every t.v.s. $E$ conditions (a), (d), (e) are equivalent.

(ii) If $E$ is a Baire space and $U$ is connected, then conditions (a), (b), (c), (d) and (e) are equivalent.

Proof. (i) Implications (e) $\Rightarrow(d),(d) \Rightarrow$ (a) are obvious. Implication (a) $\Rightarrow(e)$ is a direct consequence of Corollary 6.1.

(ii) By (i) it is enough to show that (b) $\Rightarrow(d)$, i.e. to prove that for every $q \in \Gamma(F)$ the set

$$
U_{0}=\{a \in U: q \circ f \text { is bounded in a neighborhood of } a\}
$$

is identical with $U$. The proof will be done if we check that condition (C) of Lemma 6.1 is satisfied.

Let $x \in U_{0}$ and $x+W \subset U$, where $W$ is a balanced neighborhood of $0 \epsilon E$. Then $f(x+h)=\sum_{n=0}^{\infty} f_{n}(h)$ if $h \epsilon W$, where $n ! f_{n}=\delta_{x}^{n} f$. By Corollary 6.1 the polynomials $f_{n}$ are $q$-continuous, by Remark 5.1 the function $f$ is $q$-continuous in $W$. Thus (C) is satisfied. The proof is concluded.

Example 6.1. The function $\Psi: l^{2} \rightarrow C$ defined by

$$
\Psi(x)=\sum_{n=0}^{\infty} n x_{n}^{n}, \quad x=\left(x_{1}, x_{2}, \ldots\right) \epsilon l^{2}
$$


is analytic in $l^{2}$, unbounded on the unit sphere. The function $f=1,1$, $\left.\Psi_{2}, \ldots\right): l^{2} \rightarrow C^{\mathbf{N}}$, where $\Psi_{k}(x)=\Psi(k x)$, is analytic but it is not locally bounded (it is unbounded in every neighborhood of $0 \in l^{2}$ ).

COROLLARY 6.2 (Hartogs theorem). Let $U$ be an open subset of $E_{1} \times E_{2}$, where at least one of the t.v.s. $E_{1}, E_{2}$ is metrizable and $E_{1} \times E_{2}$ is a Baire space (so both $E_{1}$ and $E_{2}$ are Baire spaces). If $f: U_{\ni}\left(x_{1}, x_{2}\right) \rightarrow f\left(x_{1}, x_{2}\right) \in F$ is separately analytic, then $f$ is analytic.

Proof. Given $x_{j}, h_{j} \in E_{j}, z_{j} \in C \quad(j=1,2)$ and $u \in F^{\prime}$, the function $u \circ f\left(x_{1}+z_{1} h_{1}, x_{2}+z_{2} h_{2}\right)$ is analytic with respect to $z_{1}$ and $z_{2}$, separately. Therefore, by the classical Hartogs theorem, the function $u \circ f\left(x_{1}+t h_{1}\right.$, $x_{2}+t h_{2}$ ) is analytic with respect to $t$ ranging a suitable open subset of $\boldsymbol{C}$. Thus it follows from Theorem 3.1 and Proposition 5.5 that $f$ is $G$-analytic. By Lemma 3, [6], for every $q \epsilon \Gamma(F)$ there exists an open subset $W_{q} \subset U$ on which $q \circ f$ is bounded. Consequently we may apply Theorem 6.1 (ii), because without loss of generality we may assume that $U$ is connected. Thus $f$ is analytic.

Observe that Theorem 6.1 (ii) implies the following

COROLLARY 6.3 (Generalization of Rado theorem). If $U$ is an open subset of a Baire space $E, f: U \rightarrow C$ is a function such that $f \mid U \cap V$ is continuous for every affine line $V \subset E$, the set $f^{-1}(0)$ is closed and $f \mid f^{-1}(\boldsymbol{C} \backslash\{0\})$ is analytic, then $f$ is analytic.

Further theorems on removable singularities will be given in our forthcoming paper.

THEOREM 6.2. Let $\boldsymbol{E}$ be an arbitrary t.v.s. over $\boldsymbol{C}$. Given any function $f: U \rightarrow F^{n}$ the following conditions are equivalent:

(i) $f$ is analytic.

(ii) $f$ is continuous and weakly analytic.

(iii) $f$ is continuous and analytic on affine lines.

(iv) $f$ is analytic on affine lines and for every $q \in \Gamma(F)$ the function $q \circ f$ is locally bounded.

Proof. Implications (i) $\Rightarrow$ (ii) and (iii) $\Rightarrow$ (iv) are obvious. If follows from Theorem 3.1 that (ii) $\Rightarrow$ (iii). If (iv) is satisfied, then $f$ is $G$-analytic because of Proposition 5.5. Thus by Theorem 6.1, we have (iv) $\Rightarrow$ (i). This concludes the proof.

THEOREM 6.3. If $E$ is metrizable, then $f: U \rightarrow F$ is analytic if and only if it is weakly-analytic.

Proof. Necessity is obvious.

Sufficiency. By Theorem 3.1 and Proposition 5.5 the function $f$ is $G$-analytic. According to Theorem 6.1 it remains to prove that for every $q \in \Gamma\left(F^{\prime}\right)$ the function $q \circ f$ is locally bounded in $U$. If it is not true, there exist $x_{0} \in U, q \in \Gamma\left(F^{\prime}\right)$ and a sequence $\left\{x_{n}\right\}_{n \in \boldsymbol{N}}$ convergent to $x_{0}$, such th. $q \circ f\left(x_{n}\right) \geqslant n, n=1,2, \ldots$ This is, however, impossible, because $\left\{f\left(x_{n}\right)\right\}_{n \in \mathbf{N}}$ is bounded, as it is weakly bounded.

Proposttion 6.2 (Vitali theorem). Let $U$ be an open connected subset. of $E$. If $f_{n}: U \rightarrow F(n \geqslant 1)$ is a sequence of analytic functions such that for every $q \in \Gamma\left(F^{\prime}\right)$ the sequence $\left\{q \circ f_{n}\right\}_{n \in \mathbf{X}}$ is locally uniformly bounded and for each $x$ belonging to an open subset $\nabla \subset U$ the sequence $\left\{f_{n}(x)\right\}_{n \in \mathbf{N}}$ is convergent to an element of $F$, then there is an analytic function $f: U \rightarrow F$ such that for every $q \in \Gamma\left(F^{\prime}\right)$

$$
q\left(f_{n}(x)-f(x)\right) \rightarrow 0 \quad(n \rightarrow \infty, x \in U),
$$

convergence being uniform on every compact subset of $U$.

Proof. a. At first we shall prove that $\left\{f_{k}\right\}_{k \in \mathbf{N}}$ converges to a function $f$ which is $G$-analytic in $U$. Put

$$
U_{0}=\left\{a \epsilon U:\left\{f_{n}(x)\right\}_{n \in \boldsymbol{N}} \text { converges for every } x\right.
$$$$
\text { belonging to a neighborhood of } a\} \text {. }
$$

In order to prove that $U_{0}=U$ let $a$ be a fixed point of $U_{0}$ and let $W$ be a balanced neighborhood of $0 \in E$ such that $a+W \subset U$. By. Proposition 3.2 the sequence of the functions $\left\{f_{n}(a+\lambda x)\right\}_{n \in \mathbf{N}}$ of one complex variable $\lambda$ is locally uniformly convergent in $|\lambda| \leqslant 1+\delta(\delta=\delta(x)>0$ sufficiently small) to an analytic function $f(a+\lambda x)$.

In particular, $\left\{f_{n}(x)\right\}_{n \in \boldsymbol{N}}$ converges for every $x \epsilon a+W$. By Lemma 6.1 , $U_{0}=U$ and by Proposition 5.5, $f=\lim f_{n}$ is $G$-analytic.

b. Given any $q \in \Gamma\left(F^{\prime}\right)$, by Proposition 6.1 the functions $f_{n}, n=1,2, \ldots$, are $q$-equicontinuous at every point $a \epsilon U$.

c. Now we shall prove that the limit function $f$ is continuous and therefore analytic (because it is $G$-analytic). Indeed, observe that for any fixed $q \epsilon \Gamma(F)$ we have

(1) $q(f(x)-f(a)) \leqslant q\left(f(x)-f_{n}(x)\right)+q\left(f_{n}(x)-f_{n}(a)\right)+q\left(f_{n}(a)-f(a)\right)$ or $x, a \in U, n=1,2, \ldots$

Given $\varepsilon>0$, let $\nabla_{a}$ be a neighborhood of $a$ such that

(2) $q\left(f_{n}(x)-f_{n}(a)\right)<\varepsilon / 9, x \in V_{a}, n=1,2, \ldots$

It follows that

(3) $q\left(f_{k}(x)-f_{l}(x)\right) \leqslant q\left(f_{k}(x)-f_{k}(a)\right)+q\left(f_{k}(a)-f_{l}(a)\right)+q\left(f_{l}(a)-f_{l}(x)\right)$ $\leqslant \varepsilon / 3, x \in V_{a}$, if $k, l \geqslant k_{0}=k_{0}(\varepsilon, a)$.

Consequently, letting $l$ tend to $\infty$, we get

$$
q\left(f_{n}(x)-f(x)\right) \leqslant \varepsilon / 3, \quad x \in V_{a}, n \geqslant k_{0} .
$$

Hence, by (1) and (2), we have

$$
q(f(x)-f(a)) \leqslant \varepsilon, \quad x \in \nabla_{a} .
$$


d. It remains to prove that the sequence $\left\{q \circ\left(f_{n}-f\right)\right\}_{n \in \mathbf{N}}$ converges to 0 uniformly on every compact subset of $U$. This is, however, a direct consequence of $a, b, c$ and of the inequality

$$
q\left(f_{n}(x)-f(x)\right) \leqslant q\left(f_{n}(x)-f_{n}(a)\right)+q\left(f_{n}(a)-f(a)\right)+q(f(a)-f(x)),
$$

which is valid for $x, a \epsilon U, n \geqslant 1$.

THEOREM 6.4 (On superposition of complex analytic functions). Let $U_{j}$ be an open subset of t.v.s. $E_{j}$ over $C(j=1,2)$. Assume that $f_{1}: U_{1} \rightarrow E_{2}$, $f_{2}: U_{2} \rightarrow F$ are analytic functions and $f_{1}\left(U_{1}\right) \subset U_{2}$. Ther $f=f_{2} \circ f_{1}$ is analytic.

Proof. By Theorems 6.2 and 3.1 it is enough to show that for every $u \in F^{\prime}$ and for every affine line $V \subset E_{1}$ the function $u \circ f \mid U_{1} \cap V$ is analytic. We shall prove this in two steps.

$1^{0}$ If $f_{k}: E_{2} \rightarrow C$ is a homogeneous polynomial of degree $k$ and $g: D \rightarrow E_{2}(D \subset C)$ is analytic, then $f_{k} \circ g$ is analytic.

Indeed, if $h=f_{k} \circ g$ and $a \in D$, then

$$
\begin{aligned}
& \frac{h(z)-h(a)}{z-a}=\frac{1}{z-a}\left(\bar{f}_{k}(g(z), \ldots, g(z))-\bar{f}_{k}(g(a), \ldots, g(a))\right. \\
& =\bar{f}_{k}\left(\frac{g(z)-g(a)}{z-a}, g(z), \ldots, g(z)\right)+\bar{f}_{k}\left(g(a), \frac{g(z)-g(a)}{z-a}, g(z), \ldots, g(z)\right)+ \\
& +\ldots+\bar{f}_{k}\left(g(a), \ldots g(a), \frac{g(z)-g(a)}{z-a}\right),
\end{aligned}
$$

whence

$$
\begin{array}{r}
h^{\prime}(a)=\lim _{z \rightarrow a} \frac{h(z)-h(a)}{z-a}=\bar{f}_{k}\left(g^{\prime}(a), g(a), \ldots, g(a)\right)+\bar{f}_{k}\left(g(a), g^{\prime}(a),\right. \\
g(a), \ldots, g(a))+\ldots+\bar{f}_{k}\left(g(a), \ldots, g(a), g^{\prime}(a)\right) .
\end{array}
$$

Thus, by Theorem 3.1, $f_{k} \circ g$ is analytic.

$2^{\circ}$ In order to end the proof it is enough to show that if $f: U \rightarrow C$ $\left(U \subset E_{2}\right)$ and $g: D \rightarrow U(D \subset C)$ are analytic, then $f \circ g$ is analytic.

Let $a \in D, b=g(a)$. Put $f_{n}=\frac{1}{n !} \delta_{b}^{n} f$. Since $f$ is locally bounded, there exists a balanced neighbourhood $W$ of $0 \in E_{2}$ such that

and

$$
\left|f_{n}(x)\right| \leqslant M 2^{-n}, \quad x \in W, n \geqslant 1,
$$

$$
f(b+x)=\sum_{n=0}^{\infty} f_{n}(x), \quad x \in W .
$$

Let $r>0$ be so small that $g(z)-g(a) \epsilon W$, when $|z-a|<r$. Then

$$
\mid f_{n}\left(g(z)-g(a)\left|\leqslant M 2^{-n}, \quad\right| z-a \mid \leqslant r, n \geqslant 1 .\right.
$$

Therefore

$$
\dot{f}(g(z))=\sum_{n=0}^{\infty} f_{n}(g(z)-g(a)), \quad|z-a|<r,
$$

the series being uniformly convergent. By $1^{\circ}$ and by Proposition 3.1, the function $f \circ g$ is analytic in $|z-a|<r$.

Proposition 6.3. Let $U$ be an open subset of $E$. Assume that a function $g: U \times[a, b] \ni(x, t) \rightarrow g(x, t) \epsilon F$ satisfies the following conditions:

(i) For any fixed $x \in U, g$ is continuous with respect to $t \in[a, b]$, and for every $q \in \Gamma(F) q \circ g$ is locally bounded in $U \times[a, b]$.

(ii) For any fixed $t \epsilon[a, b], g$ is analytic with respect to $x \in U$.

Then the function

$$
f(x)=\int_{a}^{b} g(x, t) d t, \quad x \in U
$$

is analytic in $U$.

Pro of. By Definition 1.1, $f(x)=\lim _{n \rightarrow \infty} f_{n}(x), x \in U$, where

$$
f_{n}(x)=\frac{b-a}{n} \sum_{s=1}^{n} g\left(x, a+\frac{s}{n}(b-a)\right), \quad x \in U, n \geqslant 1 .
$$

It is obvious that $f_{n}$ is analytic in $U$. Moreover, given $q \epsilon \Gamma(F)$ and $x_{0} \in U$ we mary find a neighborhood $W$ of $x_{0}, W \subset U$, such that

whence

$$
q \circ g(x, t) \leqslant M_{q}, \quad x \in W, t \in[a, b],
$$

$$
q \circ f_{n}(x) \leqslant M_{q}(b-a), \quad x \in W, n \geqslant 1 .
$$

By Vitali theorem (Proposition 6.2) the function $f$ is analytic.

Propostrion 6.4. If $f: U \rightarrow F$ is analytic, then for every $k=1,2, \ldots$ the function.

is analytic.

$$
U \times E \ni(x, y) \rightarrow \delta_{x}^{k} f(y) \in F
$$

Proof. Given $\left(x_{0}, y_{0}\right) \in U \times E$, let $W$ be a balanced neighborhood of $0 \epsilon E$ such that $x_{0}+W+W \subset U$ and let $r$ be so small that $r y_{0} \epsilon W$. There exists a neighborhood $V$ of $y_{0}$ such that $r V \subset W$, and consequently $t V \subset W$, if $|t|=r$. Thus

$$
\delta_{x}^{k} f(y)=\frac{k !}{2 \pi i} \int_{|l|=r} f(x+t y) t^{-k-1} d t, \quad x \in x_{0}+W, y \in V .
$$

The function under the integral is analytic with respect to $(x, y)$ (Theorem 6.4) and continuous with respect to $(x, y, t)$. Thus we may apply Proposition 6.3, whence the required result follows. 
Proposition 6.5. If $f_{n}: U \rightarrow F^{\prime}(n=1,2, \ldots)$ is analytic and $f: U \rightarrow E^{\prime}$ is a function such that for every $q \in \Gamma\left(F^{\prime}\right)$ the sequence $\left\{q \circ\left(f_{n}-f\right)\right\}_{n \in \mathbf{N}}$ converges to 0 locally uniformly in $U$, then

$1^{0} f$ is analytic,

$2^{0} q\left(\delta_{x}^{k} f_{n}(y)-\delta_{x}^{k} f(y)\right) \rightarrow 0$ (if $n \rightarrow \infty$ ) locally uniformly in $U \times E$ for every $q \epsilon \Gamma(F)$ and $k \in \boldsymbol{N}$.

Proof. $1^{\circ}$ It follows from the assumptions that $f$ is continuous in $U$ and analytic on affine lines. Thus $f$ is analytic in $U$.

$2^{\circ}$ Given $x_{0} \epsilon U$ let $W$ be a neighborhood of $0 \epsilon E$ such that $x_{0}+W \subset U$ and $\varepsilon_{n}=\sup \left\{q\left(f_{n}\left(x_{0}+x\right)-f\left(x_{0}+x\right)\right): x \in W\right\} \rightarrow 0$. Let $V$ be a balanced neighbourhood of $0 \in E$ such that $V+V \subset W$. Since

$\delta_{x}^{k} f_{n}(y)-\delta_{x}^{k} f(y)=\frac{k !}{2 \pi i} \int_{|t|=1}\left(f_{n}(x+t y)-f(x+t y)\right) t^{-k-1} d t, \quad x \in x_{0}+V, y \in V$, so

$$
q\left(\delta_{x}^{k}\left(f_{n}(y)-f(y)\right)\right) \leqslant k ! \varepsilon_{n}, \quad x \in x_{0}+V, y \in V
$$

Let $y_{0}$ be any fixed point of $E$. Let $\lambda>0$ be so small that $\lambda y_{0} \in V$. The mapping $\Phi: E \ni x \rightarrow \lambda^{-1} x \epsilon E$ is a homeomorphism. Thus $\Phi(V)$ is a neighborhood of $y_{0}$. Of course

$$
q\left(\delta_{x}^{k} g_{n}(\lambda y)\right)=\lambda^{k} q\left(\delta_{x}^{k} g_{n}(y)\right) \leqslant k ! \varepsilon_{n},
$$

if $y \in \Phi(V), x \in x_{0}+V$, where $g_{n}=f_{n}-f$. Therefore

$$
q\left(\delta_{x}^{k} g_{n}(y)\right) \leqslant k ! \lambda^{-k} \varepsilon_{n}, \quad y \in \Phi(V), x \in x_{0}+V, n=1,2, \ldots
$$

The proof is concluded.

Proposimion 6.6 (Identity theorems). I. Let $U$ be an open connected subset of t.v.s. $E$ over $K$. If $f: U \rightarrow F$ is analytic and $f(x)=0$ for $x$ in an open subset $V \subset U$, then $f=0$.

II. Let $U$ be an open connected subset of the complexification $\tilde{E}$ of a t.v.s. $E$ over $\boldsymbol{R}$. If $f: \tilde{U} \rightarrow F$ is analytic, $\tilde{U} \subset \tilde{E}$ contains an open subset $V \subset E$ and $f(x)=0$ for $x \in \bar{V}$, then $f=0$.

Proof. I. Put $U_{0}=\{a \in U: f(x)=0$ in a neighborhood of $a\}$ and check that $U$ and $U_{0}$ satisfy condition (C) of Lemma 6.1. Thus $U_{0}=U$.

II. Let $a$ be a fixed point of $V$ and let $\tilde{W} \subset \tilde{E}$ be a balanced neighborhood of $0 \epsilon \tilde{E}$ such that $a+\tilde{W} \subset \tilde{U}$. Let $W \subset E$ be a neighborhood of $0 \in E$ such that $a+W \subset V$ and $W+i W \subset \tilde{W}$. Then by the analyticity of $f$,

$$
f(a+x+i y)=\sum_{n=0}^{\infty} f_{n}(x+i y), \quad x+i y \in W+i W
$$

where $n ! f_{n}=\delta_{a}^{n} f$. It is obvious that $\left(\delta_{a}^{n} f\right) \mid E=\delta_{a}^{n}(f \mid V)$. Therefore $f=0$ in $a+W$. Hence $f_{n}=0$ in $E$. This, by Theorem 3, [6], implies that $f_{n}=0$ in $\tilde{E}$. Thus $f=0$ in $a+W+i W$. Finally, by $\mathrm{I}, f=0$ in $\tilde{U}$.

Propostrton 6.7 (Maximum property). If $f: U \rightarrow F$ is analytic and $q$ is any continuous seminorm in $F$, then $q$ of cannot attain its local maximum at any interior point a of $U$, unless $q \circ f$ is constant in a neighbourhood of $a$.

Proof. By Corollary 3.3 the function $q \circ f \mid U \cap V$ is subharmonic for every affine line $V \subset E$. Thus $q \circ f$ satisfies the maximum property.

7. Analytic functions in topological vector spaces over $\mathbf{R}$. In this section all t.v.s. are over $\boldsymbol{R} ; U$ is an open subset of t.v.s. $E$.

THEOREM 7.1. Assume that $E$ is a t.v.s. over $\boldsymbol{R}$ such that its complexification $\tilde{E}$ is a Baire space (this assumption is certainly satisfied if $E$ is a Fréchet space). Then for any analytic function $f: U \rightarrow F$ one may find an open subset $\tilde{U}$ of $\tilde{E}$ and an analytic function $\tilde{f}: \tilde{U} \rightarrow \tilde{F}$ such that $U \subset \tilde{U}$ and $\tilde{f} \mid U=f$.

Proof. For every point $a \in U$ there exists a balanced neighborhood $W_{a}$ of $0 \epsilon E$ such that

$$
f(a+x)=\sum_{n=0}^{\infty} f_{n}(x), \quad x \in W_{a}+W_{a}
$$

where $f_{n}=\frac{1}{n !} \delta_{a}^{n} f$. By Lemma 4 a, [6], the complexified series $\sum_{n=0}^{\infty} \tilde{f}_{n}$ converges in $\frac{1}{4 e}\left(W_{a}+i W_{a}\right)$. Since $\tilde{E}$ is a Baire space, we may apply Theorem 5.2. Thus the function

$$
\tilde{f}_{a}(x)=\sum_{n=0}^{\infty} \tilde{f}_{n}(x-a), \quad x \epsilon a+\frac{1}{4 e}\left(W_{a}+i W_{a}\right)
$$

is analytic in $V_{a}=a+U_{a}+i U_{a}$, where $U_{a}=\frac{1}{4 e} W_{a}$. It is obvious that $\tilde{f}_{a}(x)=f(x)$ for $x \in a+U_{a}$. The set $\tilde{U}=\bigcup_{a \in U} V_{a}$ is open in $\tilde{E}$ and $U \subset \tilde{U}$. The proof will be completed if we show that $\tilde{f}_{a}(x)=\tilde{f}_{b}(x)$ in $V_{a} \cap V_{b}$, because then the required function $\tilde{f}$ is given by

$$
\tilde{f}(x)=\tilde{f}_{a}(x), \quad x \in V_{a}, a \in U .
$$

Observe that $V_{a} \cap V_{b}=\left(a+U_{a}\right) \cap\left(b+U_{b}\right)+i\left(U_{a} \cap U_{b}\right)$. Without loss of generality we may assume that $V_{a} \cap V_{b}$ is connected or equivalently that $\left(a+U_{a}\right) \cap\left(b+U_{b}\right)$ is connected. Since $\tilde{f}_{a}-\tilde{f}_{b}=0$ in $\left(a+U_{a}\right) \cap$ $\cap\left(b+U_{b}\right)$, we may apply Proposition 6.6, and therefore $\tilde{f}_{a}=\tilde{f}_{b}$ in $V_{a} \cap V_{b}$. The proof is concluded. 
THEOREM 7.2. Assume that $E$ is a Baire space and $F$ is a Banach space. Then for any analytic function $f: U \rightarrow F$ one may find an open subset $\tilde{U}$ of $\tilde{E}$ and an analytic function $\tilde{f}: \tilde{U} \rightarrow \tilde{F}$ such that $U \subset \tilde{U}$ and $\tilde{f} \mid U=f$.

Proof. It follows from the proof of the previous theorem that it is enough to show that for every point $a \in U$ there is a neighborhood $V_{a} \subset \tilde{E}$ of $a$ and an analytic function $\tilde{f}_{a}: V_{a} \rightarrow \tilde{F}$ such that $\tilde{f}_{a}\left|V_{a} \cap U=f\right| V_{a} \cap U$.

Since $E$ is a Baire space we may assume, by Proposition 5.2, that $\sum_{n=0}^{\infty} \sup \left\{q \circ f_{n}(x): x \in W_{a}+W_{a}\right\}<\infty$, where $\sum_{n=0}^{\infty} f_{n}$ denotes the formal series of $(*)$, and $q$ the norm in $F$. Thus, by Proposition 6 a, [6],

$$
\sum_{n=0}^{\infty} \sup \left\{\tilde{q} \circ \tilde{f}_{n}(x): x \epsilon \frac{1}{4 e}\left(W_{a}+i W_{a}\right)\right\}<\infty
$$

and therefore by virtue of Proposition 6.2, the function

$$
\tilde{f}_{a}(x)=\sum_{n=0}^{\infty} \tilde{f}_{n}(x-a), \quad x \in V_{a}=a+\frac{1}{4 e}\left(W_{a}+i W_{a}\right),
$$

is analytic.

The following theorem is an immediate consequence of Theorems 7.1 and 6.4 .

THEOREM 7.3 (On superposition of real analytic functions). Let $U_{j}$ be an open subset of a t.v.s. $E_{j}$ over $\boldsymbol{R}(j=1,2)$. Assume that $\tilde{E}_{1}$ and $\tilde{E}_{2}$ are Baire spaces, $f_{1}: U_{1} \rightarrow E_{2}, f_{2}: U_{2} \rightarrow F$ are analytic functions and $f_{1}\left(U_{1}\right)$ $\subset U_{2}$. Then $f=f_{2} \circ f_{1}$ is analytic.

Lemma 7.1. Suppose $\mathbb{F}^{\prime \prime}$ (the topological dual of $F^{\prime}$ ) is a Baire space. A function $f: \boldsymbol{R} \rightarrow F$ is analytic if and only if it is weakly analytic.

Proof. By Lemma 2.1 the function $f$ is of class $C^{\infty}$. Fix $t_{0} \in \boldsymbol{R}$. It is enough to prove that the Taylor series $\sum_{n=0}^{\infty} a_{n} t^{n}, a_{n}=\frac{1}{n !} f^{(n)}\left(t_{0}\right)$, of the function $f$ at $t_{0}$, converges in a neighborhood of zero (because then the fact that $f$ is weakly analytic implies that its sum is equal to $f\left(t_{0}+t\right)$ ).

Thus it remains to show that there exists $\varrho>0$ such that for every $q \epsilon \Gamma\left(F^{\prime}\right)$ one may find $M_{q}>0$ such that

$$
q\left(a_{n}\right) \leqslant M_{q} \varrho^{-n}, \quad n=1,2, \ldots
$$

Since $F^{\prime}$ is a Baire space and the sequence of continuous functions $\left\{F^{\prime} \ni u \rightarrow\left|u\left(a_{n}\right)\right|^{1 / n} \in \boldsymbol{R}\right\}_{n \in \mathbf{N}}$ is bounded at every point $u \in F^{\prime}$, we may find an open subset $V \subset F^{\prime}$ and a positive number $M$ such that

$$
\left|u\left(a_{n}\right)\right| \leqslant M^{n}, \quad u \in V, n=1,2, \ldots
$$

This and the linearity of the mapping $F^{\prime} э u \rightarrow u\left(a_{n}\right) \in \boldsymbol{R}$ imply that there exists a neighborhood $W \subset F^{\prime}$ of zero such that

$$
\left|u\left(a_{n}\right)\right| \leqslant 2 M^{n}, \quad u \in W, n=1,2, \ldots
$$

Since $W$ is absorbing, for every $u \in F^{\prime}$ there exists $\lambda>0$ such that $\left|u\left(a_{n}\right)\right| \leqslant \lambda M^{n}(n \geqslant 1)$. Thus

$$
\limsup _{n \rightarrow \infty} \sqrt[n]{\left|u\left(a_{n}\right)\right|} \leqslant M, \quad u \epsilon F^{\prime}
$$

Therefore the radius of convergence of $\sum_{n=0}^{\infty} u\left(a_{n}\right) t^{n}$ is $\leqslant 2 \varrho=1 / M$. The Cauchy inequalities $\left|u\left(a_{n}\right)\right| \leqslant M_{u} \varrho^{-n}\left(n \geqslant 1, u \in F^{\prime}\right)$ imply that for any $q \in \Gamma\left(F^{\prime}\right)$ there exists $M_{q}>0$ such that $(*)$ is satisfied. The proof is concluded.

THEOREM 7.4. Let $U$ be an open subset of a Baire metrizable vector space $E$ over $\boldsymbol{R}$. If $F^{\prime}$ is a Baire space, then for any function $f: U \rightarrow F^{\prime}$ the following conditions are equivalent:

(a) $f$ is weakly-analytic.

(b) $f$ is analytic.

(c) The function $\Phi: F^{\prime} \times U \ni(u, x) \rightarrow u \circ f(x) \in \boldsymbol{R}$ is analytic.

Proof. (a) $\Rightarrow$ (b). Let $a$ be a fixed point of $U$. For every $x \in E$ there exists $r>0$ such that the function $(-r, r) \rightarrow t \rightarrow f(a+t x) \in F$ is analytic (Lemma 7.1). Therefore

where

$$
f(a+x)=\sum_{n=0}^{\infty} f_{n}(x), \quad x \in A
$$

$$
n ! f_{n}(x)=\left.\frac{d^{n}}{d t^{n}} f(a+t x)\right|_{t=0}
$$

and $A$ is an absorbing subset of $\boldsymbol{R}$. because

If $u \in F^{\prime}, u \circ f_{n}$ is a continuous homogeneous polynomial of degree $n$,

$$
n ! u \circ f_{n}(x)=\left.\frac{d^{n}}{d t^{n}} u \circ f(a+t x)\right|_{t=0} .
$$

This, according to Corollary 4, [6], and Proposition 5. [6], implies that $f_{n}$ is a continuous homogeneous polynomial of degree $n$.

By virtue of Proposition 5.2 it remains to prove that the series $\sum_{n=0}^{\infty} f_{n}$ converges in a neighborhood of $0 \epsilon E$. (Its sum is necessarily equal to $f$, because $\sum_{n=0}^{\infty} f_{n}$ converges weakly to $f$ ). 
Let $W_{n}$ be a balanced neighborhood of $0 \in E$, the diameter of $W_{n}$ being $<1 / n$. For each $n \in \boldsymbol{N}$ the set

$$
S_{n}=\bigcap_{x \in W_{n}^{\prime}} T_{x}, \quad \text { where } T_{x}=\left\{u \in F^{\prime}:\left|u \circ f_{k}(x)\right| \leqslant 1, k=1,2, \ldots\right\},
$$

is closed and $F^{\prime}=\bigcup_{n=0}^{\infty} S_{n}$. Indeed, for any $u \in F^{\prime}$ the series $\sum_{n=0}^{\infty} u \circ f_{n}$ is normally convergent at zero (Proposition 5.2), so there exists $n_{0}$ such that

$$
\sup \left\{\left|\varkappa \circ f_{k}(x)\right|: x \in W_{n_{0}}, k=1,2, \ldots\right\}<1 .
$$

Thus $u \in S_{n_{0}}$.

Since $F^{\prime}$ is a Baire space, there exist a neighborhood $V$ of $0 \epsilon F^{\prime}$, $u_{0} \in F^{\prime}$ and $m \in N$ such that

$$
\sup \left\{\left|u \circ f_{k}(x)\right|: k=1,2, \ldots\right\} \leqslant 1, \quad u \in u_{0}+V, x \in W_{m} .
$$

Hence $\sup \left\{\left|u \circ f_{k}(x)\right|: k=1,2, \ldots\right\} \leqslant 2$ for $u \in V, x \in W_{m}$. Since $V$ is an absorbing subset of $F^{\prime}$, this implies that for every $u \in F^{\prime}$ the sequence $\left\{u \circ f_{k}\right\}_{k \in \boldsymbol{N}}$ is uniformly bounded in $W_{m}$. Therefore the sequence $\left\{f_{k}\right\}_{k \in \boldsymbol{N}}$ is uniformly bounded in $W_{m}$. Thus the series $\sum_{k=0}^{\infty} f_{k}$ converges in $W_{m}$ and $f(a+x)=\sum_{k=0}^{\infty} f_{k}(x)$ for $x \in W_{m}$.

(b) $\Rightarrow$ (c). The analyticity of $f$ implies that for any $a \in U$ there exists a neighborhood $W$ of $0 \in E$ such that

$$
\Phi(u, a+x)=\sum_{n=0}^{\infty} u \circ f_{n}(x), \quad u \in F^{\prime}, x \in W,
$$

where $f_{n}=\frac{1}{n !} \delta_{a}^{n} f$. The function

$$
\Phi_{n}: F^{\prime} \times E \ni(u, x) \rightarrow u \circ f_{n}(x) \in \boldsymbol{R}
$$

is a homogeneous polynomial of degree $n_{k}+1$, continuous with respect to $u$ and $x$ separately. By Lemma 3, [6], and Theorem 1, [6], the polynomial $\Phi_{n}$ is continuous. It is obvious that

$$
\Phi\left(u_{0}+u, a+x\right)=\Phi\left(u_{0}, a\right)+\sum_{n=1}^{\infty} \Phi_{n}\left(u_{0}, x\right)+\Phi_{n-1}(u, x), \quad u \in F^{\prime}, x \in W
$$

whence it follows that $\Phi$ is analytic, because $\Phi_{n}\left(u_{0}, x\right)+\Phi_{n-1}(u, x)$ is a continuous homogeneous polynomial of degree $n$ from $F^{\prime} \times E$ to $\boldsymbol{R}$.

(c) $\Rightarrow(\mathrm{a})$ is a direct consequence of the following

LEMLA 7.2. Let $E_{j}(j=1,2)$ and $F$ be t.v.s. over $K$. If $U_{j}$ is an open subset of $E_{j}$ and $f: U_{1} \times{U_{2}}{ }(x, y) \rightarrow(x, y) \in F$ is analytic, then $f$ is analytic with respect to $x$ and $y$, seperately.
Proof. Given $(a, b) \epsilon U_{1} \times U_{2}, f(a+x, b+y)=\sum_{k=0}^{\infty} f_{k}(x, y)$ for $(x, y)$ $\epsilon W_{1} \times W_{2}$, where $W_{j}$ is a neighborhood of $0 \epsilon E_{j}$ and $f_{k}$ is a continuous homogeneous polynomial of degree $k$ from $E_{1} \times E_{2}$ to $F$. It is obovious that $E_{1} \rightarrow x \rightarrow f_{k}(x, 0) \in F$ is a continuous homogeneous polynomial of degree $k$. Thus the continuous function $U_{1}{ }^{3} x \rightarrow f(x, b) \epsilon F$ is analytic for each $b \in U_{2}$, because

$$
f(a+x, b)=\sum_{k=0}^{\infty} f_{k}(x, 0), \quad x \in W_{1} .
$$

From the proof of $(a) \Rightarrow(b)$ we deduce easily the following

Remark 7.1. If $E, F, U$ satisfy the assumptions of Theorem 7.4, and $f: U \rightarrow F$ is analytic, then for every $a \epsilon U$ there exists a balanced neighborhood $W$ of $0 \in E$ such that

$$
f(a+x)=\sum_{n=0}^{\infty} f_{n}(x), \quad x \in W
$$

where $f_{n}=\frac{1}{n !} \delta_{a}^{n} f$, and, moreover, for every $q \in \Gamma(F)$

$$
\sum_{n=0}^{\infty} \sup \left\{q \circ f_{n}(x): x \in W\right\}<\infty \text {. }
$$

Hence, by Proposition 6a, [6], and Proposition 6.2, the function $f$ may be extended to an analytic function $\tilde{f}: \tilde{U} \rightarrow \tilde{F}$, where $\tilde{U}$ is an open subset of $\tilde{E}$ such that $U \subset \tilde{U}$.

THEOREM 7.5. Let $U$ be an open subset of a real Baire space $E$. If $F^{\prime}$ is a metrizable space (e.g. if $F$ is Banach space), then $f: U \rightarrow F$ is analytic if and only if $f \in G^{\infty}(U), f$ is continuous and $f \mid U \cap V$ is analytic for every affine line $V \subset E$.

Proof. The definition of $G^{\alpha}(U)$ implies that all the Gateaux differentials $\delta_{x}^{n} f(n \geqslant 1)$ exist, and the analyticity on affine lines implies that

$$
f(x+h)=\sum_{n=0}^{\infty} f_{n}(h), \quad h \in A
$$

where $f_{n}=\frac{1}{n !} \delta_{x}^{n} f$ and $A$ is an absorbing set.

By Theorem 5, [6], the polynomials $f_{n}$ are continuous. The sets

$$
A_{k l}=\left\{x \in E:||_{n} f_{n}(x) \mid \leqslant k, \text { dist }(u, 0) \leqslant \frac{1}{l}, u \in F^{\prime}, n \geqslant 1\right\}
$$


are closed, $A \subset \bigcup_{k, l=1}^{\infty} A_{k l}$ and $E=\bigcup_{s, k, l=1}^{\infty} s A_{k l}$. So there exists $k, l$ such that int $A_{k l} \neq \varnothing$. Therefore

$$
\left|u \circ f_{n}(x)\right| \leqslant M_{u}, \quad u \in F^{\prime}, n \geqslant 1, x \in A_{k l},
$$

where, by virtue of Lemma 2, [6]

$$
q \circ f_{n}(x) \leqslant M_{q}, \quad q \in \Gamma(F), n \geqslant 1, x \in W,
$$

where $W$ is a neighborhood of $0 \epsilon E$.

This implies that $\sum_{n=0}^{\infty} f_{n}$ converges in a neighborhood $W$ of $0 \epsilon \mathcal{E}$. By the analyticity on affine lines

$$
f(x+h)=\sum_{n=0}^{\infty} f_{n}(h), \quad h \epsilon W .
$$

From Theorems 7.4 and 7.5 we deduce easily the following

CoROLlaRy 7.1. Let $U$ be an open subset of a Baire metrizable space $E$. If $F^{\prime}$ is a Baire space, then $f: U \rightarrow F$ is analytic if and only if $f \epsilon G^{\infty}(U)$, $f$ is weakly continuous and $f \mid U \cap V$ is analytic for every affine line $V \subset E$.

In the following Corollaries $E$ is a Baire space, $F$ is a Banach space and $U$ is an open subset of $E$.

CoROLLARY 7.2. If $f: U \rightarrow \boldsymbol{R}$ is a continuous function of class $G^{\infty}$ such that $\delta_{x}^{n} f(a) \geqslant 0$ for $x \in U, a \in A, n \geqslant 1$, where $A$ is a subset of $E$ such that $\bigcup_{a \in A} \boldsymbol{R} a=E$, then $f$ is analytic.

Corollary 7.3. If $f: U \times \boldsymbol{R} \rightarrow \boldsymbol{R}$ is analytic, $f \neq 0, \varphi: U \rightarrow \boldsymbol{R}$ is continuous, $f(x, \varphi(x)) \equiv 0$ and $\varphi \in G^{\infty}(U)$, then $\varphi$ is analytic.

Corollaries 7.2 and 7.3 may be proved analogously as in [4].

COROLLARY 7.4. A function $f: U \rightarrow F$ is analytic if and only if it is continuous and weakly-analytic.

Proof. By Theorem 7.4 the function $f \mid U \cap V$ is analytic for every vector subspace $V \subset E, \operatorname{dim} V<\infty$. Hence, by Theorem 4, [6], $f \in G^{\infty}(U)$. We may apply Theorem 7.5.

COROLLARY 7.5. A function $f: U \rightarrow F$ is analytic if and only if for each $a \in U$ there exist a neighborhood $W$ of a, a sequence of continuous polynomials $g_{n}: E \rightarrow F\left(\operatorname{deg} g_{n} \leqslant n\right), n=1,2, \ldots$, and constants $a>0,1>\beta$ $>0$ such that

$$
\left\|f(y)-g_{n}(y)\right\| \leqslant \alpha \beta^{n} \quad \text { for } y \in W .
$$

Pro of. The necessity follows from Proposition 5.1. In order to prove sufficiency, we may assume that $\dot{F}=\boldsymbol{R}$ (Corollary 7.4).
Let $m$ be any fixed positive integer and let $r>0$ be so small that the set

$$
W_{m}=\left\{a+t_{1} x_{1}+\ldots+t_{m} x_{m}: t_{j} \in \boldsymbol{R},\left|t_{j}\right| \leqslant r(j=1, \ldots, m)\right\}
$$

is contained in $W$, where $x_{1}, \ldots, x_{m}$ are any fixed elements of $E$. It follows from (*) that

$$
\left|g_{n+1}(x)-g_{n}(x)\right| \leqslant \alpha(\beta+1) \beta^{n}, \quad x \in W_{m}, n \geqslant 1 .
$$

Let $\omega$ satisfy the inequalities $1<\omega<1 / \beta$. It follows from (**) and from the Polynomial Lemma that

for

$$
\left|\tilde{g}_{n+1}(x)-\tilde{g}_{n}(x)\right| \leqslant M \alpha(\beta+1) \omega(\beta \omega)^{n}, \quad n \geqslant 1,
$$

$$
\begin{array}{r}
x \in \tilde{W}_{m}=\left\{a+\left(t_{1}+i \tau_{1}\right) x_{1}+\ldots+\left(t_{m}+i \tau_{m}\right) x_{m}: t_{j}, \tau_{j} \in \boldsymbol{R},\left|t_{j}\right| \leqslant r,\left|\tau_{j}\right|<\delta,\right. \\
j=1, \ldots, m\},
\end{array}
$$

$\tilde{g}_{j}$ denoting the complexification of $g_{j}$. Therefore the function

$$
\tilde{g}(x)=\tilde{g}_{1}(x)+\sum_{j=1}^{\infty}\left(\tilde{g}_{j+1}(x)-\tilde{g}_{j}(x)\right), \quad x \in \tilde{W}_{m},
$$

is analytic in $\tilde{W}_{m}$. In particular, $\tilde{g}\left|W_{m}=f\right| W_{m}$ is analytic. Thus (by Theorem 5, [6]) $f \epsilon G^{\infty}(U)$ and $f \mid U \cap V$ is analytic for any affine line $V \subset E$.

CoROLLARY 7.6. A function $f: U \rightarrow F$ is analytic if and only if $f \epsilon G^{\infty}(U)$, $f$ is continuous and for every subset $I=\{a+t(b-a): 0 \leqslant t \leqslant 1\} \subset U$ there exists an absorbing subset $A \subset E$ such that for every $x \in I$ the series $\sum_{n=0}^{\infty} \frac{1}{n !} \delta_{x}^{n} f$ converges in $A$.

Proof. By the Pringsheim-Boas theorem, [3], $f \mid I$ is analytic. Thus we may apply Theorem 7.5.

\section{Entire functions.}

Definition 8.1. Let $E, F$ be t.v.s. over $K$. A function $f: E \rightarrow F$ is called entire, if there exists a formal series $\sum_{n=0}^{\infty} f_{n}$ from $E$ to $F$ such that

$$
f(x)=\sum_{n=0}^{\infty} f_{n}(x), \quad x \in E .
$$

Proposttron 8.1. A function $f: E \rightarrow F$ is entire if and only if it is weakly entire.

Proof. It is obovious that $f$ is weakly entire if it is entire. Let

$$
u \circ f(x)=\sum_{n=0}^{\infty} g_{n}(u ; x), \quad x \in E, u \in F^{\prime},
$$


where $g_{n}(u ; \cdot) \in Q^{n}(E, K)$ for any $u \in F^{\prime \prime}$. It follows from the equation

$$
u \circ f(t: x)=\sum_{n=0}^{\infty} t^{n} g_{n}(u ; x), \quad t \in K,
$$

and from. Lemma 2.1 that

$$
n ! g_{n}(u ; x)=\left.\frac{d^{n}}{d t^{n}} u \circ f(t x)\right|_{t=0}=\left.u \circ \frac{d^{n}}{d t^{n}} f(t x)\right|_{t=0} .
$$

Thus, according to Corollary 4, [6], $\left.\frac{d^{n}}{d t^{n}} f(t x)\right|_{t=0}$ is a homogeneous polynomial of degree $n$ and by Proposition 5.6

$$
f(x)=\sum_{n=0}^{\infty} \frac{1}{n !} \delta_{0}^{n} f(x), \quad x \in E .
$$

Theorems 5.1, 5.2 and Remark 5.1 imply the following

Proposition 8.2. $1^{\circ}$ If $E$ and $F$ are t.v.s. over $\boldsymbol{R}$, then every entire function $f: E \rightarrow F$ may be continued to entire function $\tilde{f}: \tilde{E} \rightarrow \tilde{F}$.

$2^{\circ}$ If $f: E \rightarrow F$ is an entire function, then $f$ is $G$-analytic in $E$ and

$$
f(a+x)=\sum_{n=0}^{\infty} \frac{1}{n !} \delta_{a}^{n} f(x), \quad x \in E, a \in E .
$$

$3^{\circ}$ If $E$ is a Baire space and $f(x)=\sum_{n=0}^{\infty} f_{n}(x), x \in E$, where $\sum_{n=0}^{\infty} f_{n} \epsilon S(E, F)$, then $f$ is analytic in $E$.

$4^{\circ}$ If $E, F$ are t.v.s. over $\boldsymbol{R}$ and $E$ is a Baire space, then every analytio entire function $f: E \rightarrow F$ may be continued to an analytic entire function $\tilde{f}: \tilde{E} \rightarrow \tilde{F}$.

Theorem 8.1. Let $E, F$ be t.v.s. over $K$. Then:

(a) A function $f: E \rightarrow F$ is entire if and only if $f V$ is entire for every affine line $V \subset E$.

(b) If $E$ is a Baire space and $f: E \rightarrow F$ is continuous, then $f$ is analytic entire function if and only if $f \mid V$ is entire function for every affine line $V \subset \mathbb{}$.

Proof. (a) We know, [20], that (a) is true if $\operatorname{dim} E<\infty$. So

$$
f(x)=\sum_{n=0}^{\infty} f_{n}(x), \quad x \in E,
$$

where $f_{n} \mid W$ is a homogeneous polynomial of degree $n$ for every vector subspace $W \subset E, \operatorname{dim} W<\infty$. This implies that $f_{n} \in Q^{n}\left(E, F^{\prime}\right)$.

(b) By (a) $f$ is an entire continuous function, by Proposition $8.1 .2^{\circ} f$ is $G$-analytic. Since $f$ is continuous and $E$ is a Baire space, so the Gateaux differentials $n ! f_{n}=\delta_{0}^{n} f$ are continuous (Theorem 5, [6]). Hence, according to Theorem 5.2, $f$ is analytic.

\section{List of problems.}

$1^{\circ}$ Does Theorem 6.1 (ii) remain true without the assumption that $E$ is a Baire space?

$2^{\circ}$ Is Theorem 6.3 true, if $E$ is not metrizable?

$3^{\circ}$ Let $E$ be a Baire space. Is $E^{2}$ a Baire space?

$4^{\circ}$ Is Theorem 7.3 true for arbitrary t.v.s. $E_{1}, E_{2}$ over $\boldsymbol{R}$ ?

$5^{\circ}$ Are Lemma 7.1 and Theorem 7.5 true, if $F$ is an arbitrary topological vector space over $\boldsymbol{R}$ ?

$6^{\circ}$ Is it enough to assume that $f$ in Theorem 7.5 is locally bounded instead of that $f$ is continuous? true?

$7^{\circ}$ Under what weaker conditions imposed on $F$, Theorem 7.5 remains

Added in proof. A. Hirschowitz gave countererexamples to the problems $1^{\circ}$ and $2^{\circ}$.

\section{References}

Some versions of the problems considered in this paper were earlier treated by many authors. In particular, the paper [1] is related to Section 2, [9] is related to Section 3 (Theorem 3.1), [13] and [21] are related to Section 4, [7], [11], [18] and [19] are related to Section $6,[2],[7],[12],[17],[23]$ are related to Section 7 and finally [16] is related to Section 8 .

[1] A. Alexiewicz and W. Orlicz, On analytic functions of real variables, Studia Math. 12 (1951), pp. 108-111.

[2] - Analytic operations in real Banach spaces, ibidem 14 (1953), pp. 57-78.

[3] R. P. Boas, A theorem on analytic functions of a real variable, Bull. Amer. Math. Soc. 41 (1935), pp. 232-236.

[4] J. Bochnak, Analytic functions in Banach spaces, Studia Math. 35 (1970), pp. 273-292.

[5] - et J. Siciak, Fonctions analytiques dans les espaces vectoriels topologiques réeles ou complexes, C. R. Ac. Sci. Paris, 270 (1970).

[6] - and J. Siciak, Polynomials and multilinear mappings in topological vector spaces, Studia Math. this fascicle, pp. 59-76.

[7] N. Bourbaki, Variétés différentielles et analytiques (Resultats) Hermann, Paris 1967.

[8] J. Dieudonné, Foundations of modern analysis, New York 1960.

[9] A. Grothendieck, Sur certains espaces de fonotions holomorphes, J. für reine und angew. Math. 192 (1953), pp. 35-54.

[10] E. Hille, Analytic function theory I, II, Boston 1962.

[11] - and E. G. Philips, Functional analysis and semigroups, Colloq. Amer. Math. Soc. 1957.

[12] J. Kurzweil, A characterization of analytic operations in real Banach spaces, Studia Math. 14 (1953), pp. 82-83. 
[13] F. Leja, Sur les suites de polynomes bornés presque partout sur la frontière d'un domain, Math. Ann. 108 (1933), pp. 517-524.

[14] P. Lelong, Seminaire P. Lelong (Analyse), 8ème année, 1967-1968, Lecture Notes 71.

[15] - Seminaire P. Lelong (Analyse), 9ème année, 1968-1969.

[16] - Fonctions et applications de type exponentiele dans les espaces vectoriels topologiques, C. R. Ac. Paris 269 (1969), pp. 420-422.

[17] B. Malgrange, Ideals of differentiable functions, Oxford 1966.

[18] Ph. Noverraz, Fonctions plurisousharmoniques et analytiques dans les espaces vectoriels topologiques complexes, Thèse, Institut H. Poincaré, 1969.

[19] J.P. Ramis, Sous-ensemble analytique d'une variété analytique banachique, Erg. d. Math. B. 53, 1970.

[20] J. Siciak, Seperately analytic functions and envelopes of holomorphy of some lower dimensional subsets of $\boldsymbol{C}^{n}$, Ann. Pol. Math. 22 (1969), pp. 145-171.

[21] - Two criteria for the continuity of the equilibrium Riesz potentials, Prace Mat. 14 (1970), pp. 91-99.

[22] - A characterization of analytic functions of $n$ real variables, Studia Math. 35 (1970), pp. 293-297.

[23] R. Welland, Bernstein's theorem for Banach spaces, Proc. Amer. Math. Soc. 19 (1968), pp. 789-792. 